\title{
Periodic homogenization of monotone multivalued operators
}

\author{
Alain Damlamian* Nicolas Meunier ${ }^{\dagger}$ and Jean Van Schaftingen ${ }^{\ddagger}$
}

24 février 2006

\begin{abstract}
Résumé
Nous étudions l'homogénéisation par la méthode de l'éclatement de Cioranescu, Damlamian et Griso (CRAS, 2002), pour des équations de la forme $-\operatorname{div} d_{\epsilon}=f$, avec $\left(\nabla u_{\epsilon}(x), d_{\epsilon}(x)\right) \in A_{\epsilon}(x)$ où $A_{\epsilon}$ est une fonction dont les valeurs sont des opérateurs maximaux monotones. Sous des hypothèses de croissance et de coercivité sur $A_{\epsilon}$, si la suite des opérateurs maximaux monotone éclatés $\left(\mathcal{T}_{\epsilon}\left(A_{\epsilon}\right)\right)(x, y)$ converge au sens des graphes vers un opérateur maximal monotone $B(x, y)$ pour presque tout $(x, y) \in \Omega \times Y$, quand $\epsilon \rightarrow 0$, alors $\left(u_{\epsilon}, d_{\epsilon}\right)$ converge faiblement dans un espace de Sobolev adéquat vers une solution $\left(u_{0}, d_{0}\right)$ de $-\operatorname{div} d_{0}=f$ et $\left(\nabla u_{0}(x), d_{0}(x)\right) \in A(x)$ où $A$ satisfait les mêmes hypothèses que $A_{\epsilon}$. Ce résultat inclut le cas où $A_{\epsilon}(x)$ est une fonction monotone continue pour presque tout $x$ dans $\Omega$.
\end{abstract}

\begin{abstract}
Using the unfolding method of Cioranescu, Damlamian and Griso (CRAS, 2002), we study the homogenization for equations of the form $-\operatorname{div} d_{\epsilon}=f$, with $\left(\nabla u_{\epsilon}(x), d_{\epsilon}(x)\right) \in A_{\epsilon}(x)$ and where $A_{\epsilon}$ is a function whose values are maximal monotone graphs. Under appropriate growth and coercivity assumptions, if the sequence of unfolded maximal monotone graphs $\left(\mathcal{T}_{\epsilon}\left(A_{\epsilon}\right)\right)(x, y)$ converges in the graphical sense to a maximal monotone graph $B(x, y)$ for almost every $(x, y) \in \Omega \times Y$, as $\epsilon \rightarrow 0$, then $\left(u_{\epsilon}, d_{\epsilon}\right)$ converges weakly in a suitable Sobolev space to a solution $\left(u_{0}, d_{0}\right)$ of the problem $-\operatorname{div} d_{0}=f$, with $\left(\nabla u_{0}(x), d_{0}(x)\right) \in$ $A(x)$ and $A$ satisfies the same assumptions as $A_{\epsilon}$. This result includes the case where $A_{\epsilon}(x)$ is a monotone continuous function for almost every $x$ in $\Omega$.
\end{abstract}

*Laboratoire d'Analyse et de Mathématiques Appliquées, CNRS UMR 8050, Université Paris 12 Val de Marne 94010 Créteil Cedex, France. (damlamian@univ-paris12.fr)

${ }^{\dagger}$ Cérémade, Université Paris Dauphine. Place du Maréchal de Lattre de Tassigny 75775 Paris, France. (meunier@ceremade.dauphine.fr)

${ }^{\ddagger}$ Département de Mathématique, Université catholique de Louvain, 2 chemin du Cyclotron, 1348 Louvain-la-Neuve, Belgique (vanschaftingen@math.ucl.ac.be). 


\section{Introduction}

This article is devoted to periodic homogenization for nonlinear partial differential equations with oscillating coefficients. This type of equation models various physical problems arising in media with holes or heterogeneous materials with various competing length-scales.

In [16], using the unfolding method [9], the periodic homogenization was considered for:

$$
\left\{\begin{array}{l}
-\operatorname{div}\left(a_{\epsilon}\left(x, \nabla u_{\epsilon}\right)\right)=f \text { in } \mathcal{D}^{\prime}(\Omega), \\
u_{\epsilon} \in W_{0}^{1, p}(\Omega),
\end{array}\right.
$$

where $\Omega$ is a Lipschitz open bounded set of $\mathbf{R}^{N}, 1<p<\infty, p^{-1}+q^{-1}=1$, $f_{\epsilon} \in W^{-1, q}(\Omega)$ and $a_{\epsilon}: \Omega \times \mathbf{R}^{N} \rightarrow \mathbf{R}^{N}$ is such that $a_{\epsilon}$ is of Carathédodory type, monotone, and has its growth controlled. If the unfolded functions $\mathcal{T}_{\epsilon}\left(a_{\epsilon}\right)$ converge almost everywhere, then the sequence of solutions $\left(u_{\epsilon}\right)_{\epsilon}$ converges, as $\epsilon \rightarrow 0$, to the solution $u_{0}$ of a problem which satisfies the same hypotheses and where $a_{0}$ only depends on the limit of $\mathcal{T}_{\epsilon}\left(a_{\epsilon}\right)$.

A natural generalization of problem (1.1) is the problem

$$
\left\{\begin{array}{l}
-\operatorname{div} d_{\epsilon}=f \text { in } \mathcal{D}^{\prime}(\Omega) \\
\left(\nabla u_{\epsilon}(x), d_{\epsilon}(x)\right) \in A_{\epsilon}(x) \\
u_{\epsilon} \in W_{0}^{1, p}(\Omega)
\end{array}\right.
$$

Now $A_{\epsilon}: \Omega \rightarrow \mathfrak{M}\left(\mathbf{R}^{N} \times \mathbf{R}^{N}\right)$ is a measurable map taking its value in the set $\mathfrak{M}\left(\mathbf{R}^{N} \times \mathbf{R}^{N}\right)$ of maximal monotone graphs from $\mathbf{R}^{N}$ to $\mathbf{R}^{N}$. If there exists $\alpha>0$ and $m \in L^{1}(\Omega)$ such that for almost every $x \in \Omega$, and for every $(\xi, \eta) \in A_{\epsilon}(x)$

$$
\alpha\left(\|\xi\|^{p}+\|\eta\|^{q}\right) \leq\langle\eta, \xi\rangle+m(x),
$$

then problem (1.2) has at least one solution [7, 13]. This solution need not be unique.

We consider here the homogenization of (1.2). Let $\left(u_{\epsilon}, d_{\epsilon}\right) \in W_{0}^{1, p}(\Omega) \times$ $L^{p}\left(\Omega ; \mathbf{R}^{N}\right)$ be solutions of the problem (1.2). If the $A_{\epsilon}$ 's satisfy assumption (1.3) uniformly and if the sequence of unfolded graphs $\mathcal{T}_{\epsilon}^{Y}\left(A_{\epsilon}\right)$ converges almost everywhere, every cluster point $\left(u_{0}, d_{0}\right)$ of the sequence $\left(u_{\epsilon}, d_{\epsilon}\right)$ for the weak topology in $W_{0}^{1, p}(\Omega) \times L^{p}\left(\Omega, \mathbf{R}^{N}\right)$, is a solution of

$$
\left\{\begin{array}{l}
-\operatorname{div} d_{0}=f \text { in } \mathcal{D}^{\prime}(\Omega), \\
\left(\nabla u_{0}(x), d_{0}(x)\right) \in A_{0}(x), \\
u_{0} \in W_{0}^{1, p}(\Omega) .
\end{array}\right.
$$

where $A_{0}: \Omega \rightarrow \mathfrak{M}\left(\mathbf{R}^{N} \times \mathbf{R}^{N}\right)$ satisfies (1.3) and is defined in terms of the limit of $\mathcal{T}_{\epsilon}^{Y}\left(A_{\epsilon}\right)$. In [16] the hypotheses were stronger and implied the strong 
convergence of the correctors, which is not obtained here. The best convergence in this direction is (4.19). If the $A_{\epsilon}$ 's satisfy additional assumptions, one recovers the strong convergence of the correctors (Theorem 4.6).

This problem is intimately connected to the convergence of maximal monotone operators, the theory of which was actively developed in the 1970's, most particularly in the Hilbert space case ([3], [1] and [2]). At the time, the first author had studied it in the case of reflexive Banach space, especially in view of the renorming result of Trojanski (keeping in mind that monotonicity is norm independent). This led to a paper which also included the case of subdifferentials of lower semi-continuous proper convex functions, but was never submitted for publication [10].

In combination with the unfolding method, a main tool here are conditions under which the local convergence of graphs implies global convergence. More precisely, denoting by $\longmapsto$ the convergence in the graphical sense, we look for conditions on $A, A^{n}: \Omega \rightarrow \mathfrak{M}\left(X \times X^{\prime}\right)$ that satisfy $A^{n}(t) \longmapsto A(t)$ under which $\mathcal{A}^{n} \longmapsto \mathcal{A}$ where

$$
\mathcal{A}=\left\{(u, v) \in L^{p}(\Omega ; X) \times L^{q}\left(\Omega ; X^{\prime}\right) \mid(u(t), v(t)) \in A(t) \text { for a.e. } t \in \Omega\right\}
$$

and a similar definition for $\mathcal{A}^{n}$.

The outline of the paper is as follows. In Section 2, we recall the definition of maximal monotone operators and of the notion of convergence of maximal monotone graphs. In section 3, using [10], we consider sequences of maximal monotone valued measurable functions, their canonical extensions and we prove key results about their convergence.

In section 4 we recall the definition of the unfolding operator, averaging operator and the corresponding convergence properties (cf. [9], [11] as well as [15], [16]).

In section 5 we consider the homogenization problem and we state our main result in Theorem 4.1.

Some of the results of this paper were announced in [16]. The case where $A_{\epsilon}(x)$ is the subdifferential of convex function with suitable growth and coercivity conditions will be presented in a forthcoming paper [12].

Note that there are many papers in the litterature under the study of $G$-convergence and homogenization which concern the non-linear case. We only refer to [8] and the bibliography therein.

\section{Maximal monotone graphs}

\section{$2.1 \quad$ Notations}

In this section we recall some basics notations about monotone and maximal monotone graphs and functions in a Banach space. For more details see $[3,4,5]$. 
Let $X$ be a reflexive Banach space and let $X^{\prime}$ be its dual. The duality product in $X^{\prime} \times X$ is denoted by $\langle\cdot, \cdot\rangle$. According to [21], there is an equivalent norm on $X$ such that both $X$ and $X^{\prime}$ are locally uniformly convex. This will be assumed from now on. It is to be noted that on a locally uniformly convex space, weak convergence together with the convergence of the norms implies strong convergence. The duality pairing associated to the norm of $X$ is denoted by $F$ and maps $\xi \in X$ to the unique $F(\xi) \in X^{\prime}$ such that $\|F(\xi)\|_{X^{\prime}}=\|\xi\|_{X}$ and $\langle F(\xi), \xi\rangle=\|\xi\|_{X}^{2}$. By the local uniform convexity of $X$ and of $X^{\prime}, F$ is an homeomorphism. Its inverse is the duality mapping from $X^{\prime}$ to $X$. In the sequel, for simplicity, $\|\cdot\|$ will denote either $\|\cdot\|_{X}$ or $\|\cdot\|_{X^{\prime}}$ since the context will make the notation clear.

We consider set-valued operators $A: X \rightarrow X^{\prime}$, that is maps which take every point $\xi \in X$ to some set $A \xi \subset X^{\prime}$. These applications are simply called operators when no confusions may arise. Similarly when no ambiguities arise, $A$ will also denote the graph of the operator $A$, that is the set $\{(\xi, \eta) \in$ $\left.X \times X^{\prime}: \eta \in A \xi\right\}$. The domain of a graph $A$ is the set,

$$
D(A)=\{x \in X \text { such that } A x \neq \emptyset\} .
$$

The operator $A$ is single-valued on some set $C \subset X$, if for every $\xi \in C$, $A \xi$ contains at most one element; it is nonexpansive if $\left\|\eta_{1}-\eta_{2}\right\| \leq\left\|\xi_{1}-\xi_{2}\right\|$ for every $\left(\xi_{1}, \eta_{1}\right),\left(\xi_{2}, \eta_{2}\right) \in A$. Every nonexpansive operator is clearly singlevalued on $X$. For operators $A, B$, we write $A \subseteq B$ whenever $A \xi \subseteq B \xi$ for every $\xi \in X$.

Monotone and maximal monotone graphs can now be defined:

Definition 2.1. The set $A \subset X \times X^{\prime}$ is a monotone graph (or monotone operator) if for every $\left(\xi_{1}, \eta_{1}\right),\left(\xi_{2}, \eta_{2}\right) \in A$,

$$
\left\langle\eta_{1}-\eta_{2}, \xi_{1}-\xi_{2}\right\rangle \geq 0
$$

The monotone graph $A$ is a maximal monotone graph (or maximal monotone operator), if for every monotone graph $B \subset X \times X^{\prime}$, the inclusion $A \subseteq B$ implies $A=B$.

Example 1. Let $A: X \rightarrow X^{\prime}$ be a single-valued map with $D(A)=X$. If $A$ is continuous and monotone then it is maximal monotone. In particular the duality mapping $F: X \rightarrow X^{\prime}$ defines a maximal monotone graph. Since $\|\cdot\|_{X}$ is strictly convex, $F$ is strictly monotone in the sense that $\langle F(x)-$ $\left.F\left(x^{\prime}\right), x-x^{\prime}\right\rangle=0$ implies $x=x^{\prime}$.

The following result plays a fundamental role in the theory of maximal monotone operators.

Theorem 2.2 (Browder [5]). Let $A \subset X \times X^{\prime}$ be a monotone graph. The graph $A$ is maximal monotone if and only if $A+F$ is a surjective map, i.e. for every $\eta \in X^{\prime}$, there is $\xi \in X$ such that $(\xi, \eta-F(\xi)) \in A$. 
Remark 1 . Since $F$ is strictly monotone, for every $\eta \in X^{\prime}$ there is at most one $\xi \in X$ such that $(\xi, \eta-F(\xi)) \in A$.

If $A$ is a maximal monotone graph, then for every $\xi \in X, A^{\xi}$ defined as $A^{\xi}=A(.+\xi)$ is also a maximal monotone graph. Hence for $\lambda>0$, there exists a unique $(\alpha, \beta) \in A^{\xi}$, i.e. $(\alpha-\xi, \beta) \in A$, such that $0=F(\alpha)+\lambda \beta$. This justifies the definition:

Definition 2.3. Let $A \subset X \times X^{\prime}$ be a maximal monotone graph. For every $\xi \in X$ and $\lambda>0,\left(J_{\lambda}^{A} \xi, A_{\lambda} \xi\right)$ denotes the unique pair in $A$ such that

$$
F\left(\xi-J_{\lambda}^{A} \xi\right)=\lambda A_{\lambda} \xi
$$

Remark 2. When $X$ is a Hilbert space, $F: X \rightarrow X^{\prime}$ is its Riesz isometric isomorphism. In that case, to every maximal monotone graph $A \subset X \times X^{\prime}$, a nonexpansive map $\phi_{A}: X \rightarrow X$ is associated such that $(\xi, \eta) \in A$ if and only if

$$
F^{-1}(\eta)-\xi=\phi_{A}\left(F^{-1}(\eta)+\xi\right) .
$$

Conversely, every nonexpansive function $\phi_{A}: X \mapsto X$ defines a maximal monotone graph in that way [17]. The maps $\phi_{A}$ and $J_{1}^{A}$ are linked as

$$
\phi_{A}(\zeta)=\zeta-2 J_{1}^{A} \zeta
$$

Proposition 2.4. The mappings $J_{\lambda}^{A}: X \rightarrow X$ and $A_{\lambda}: X \rightarrow X^{\prime}$ are continuous. The graph $A_{\lambda}$ is single-valued and maximal monotone.

The proof of Proposition 2.4 relies on the following Lemma:

Lemma 2.5. Let $A \subset X \times X^{\prime}$ be a maximal monotone operator and $\left(\xi_{n}, \eta_{n}\right) \in$ A. Suppose that, as $n \rightarrow+\infty$,

$$
\begin{aligned}
& \xi_{n} \rightarrow \xi \quad \text { weakly in } X, \\
& \eta_{n} \rightarrow \eta \quad \text { weakly in } X^{\prime}, \\
& \liminf _{n \rightarrow+\infty}\left\langle\eta_{n}, \xi_{n}\right\rangle \leq\langle\eta, \xi\rangle,
\end{aligned}
$$

then $(\xi, \eta) \in A$ and

$$
\liminf _{n \rightarrow+\infty}\left\langle\eta_{n}, \xi_{n}\right\rangle=\langle\eta, \xi\rangle .
$$

Proof. For every $(\alpha, \beta) \in A$, by the monotonicity of $A$,

$$
\left\langle\beta-\eta_{n}, \alpha-\xi_{n}\right\rangle \geq 0 \text {. }
$$

Therefore

$$
0 \leq \liminf _{n \rightarrow \infty}\left\langle\beta-\eta_{n}, \alpha-\xi_{n}\right\rangle \leq\langle\beta-\eta, \alpha-\xi\rangle .
$$

Since $A$ is maximal and $(\alpha, \beta) \in A$ is arbitrary, $(\xi, \eta) \in A$.

The fact that $(\xi, \eta) \in A$ and the monotonicity of $A$ now yield

$$
\liminf _{n \rightarrow \infty}\left\langle\eta_{n}, \xi_{n}\right\rangle \geq \lim _{n \rightarrow \infty}\left\langle\eta_{n}, \xi\right\rangle+\lim _{n \rightarrow \infty}\left\langle\eta, \xi_{n}\right\rangle-\langle\eta, \xi\rangle=\langle\eta, \xi\rangle
$$


Proof of Proposition 2.4. Let $(\alpha, \beta) \in A$. By the monotonicity of $A$, for every $\xi \in X$, we have

$$
\left\langle F\left(\xi-J_{\lambda}^{A} \xi\right), \alpha-J_{\lambda}^{A} \xi\right\rangle \leq \lambda\left\langle\beta, J_{\lambda}^{A} \xi-\alpha\right\rangle
$$

hence,

$$
\left\|\xi-J_{\lambda}^{A} \xi\right\|^{2} \leq \lambda\|\beta\|\left\|J_{\lambda}^{A} \xi-\alpha\right\|+\|\alpha-\xi\|\left\|J_{\lambda}^{A} \xi-\xi\right\|,
$$

therefore, $J_{\lambda}^{A}$ is bounded on bounded sets.

Assume $\xi_{n} \rightarrow \xi$ strongly in $X$. The monotonicity of $A$ and of $F$ imply that

$$
\begin{gathered}
\left\langle F\left(\xi_{n}-J_{\lambda}^{A} \xi_{n}\right)-F\left(\xi_{m}-J_{\lambda}^{A} \xi_{m}\right), J_{\lambda}^{A} \xi_{n}-J_{\lambda}^{A} \xi_{m}\right\rangle \geq 0 \\
\left\langle F\left(\xi_{n}-J_{\lambda}^{A} \xi_{n}\right)-F\left(\xi_{m}-J_{\lambda}^{A} \xi_{m}\right),\left(\xi_{n}-J_{\lambda}^{A} \xi_{n}\right)-\left(\xi_{m}-J_{\lambda}^{A} \xi_{m}\right)\right\rangle \geq 0 .
\end{gathered}
$$

Summing the two previous inequalities gives

$$
\left\langle F\left(\xi_{n}-J_{\lambda}^{A} \xi_{n}\right)-F\left(\xi_{m}-J_{\lambda}^{A} \xi_{m}\right), \xi_{n}-\xi_{m}\right\rangle \geq 0 .
$$

By (2.3), the left-hand side tends to 0 as $m, n \rightarrow \infty$. Consequently,

$$
\lim _{m, n \rightarrow+\infty}\left\langle F\left(\xi_{n}-J_{\lambda}^{A} \xi_{n}\right)-F\left(\xi_{m}-J_{\lambda}^{A} \xi_{m}\right), J_{\lambda}^{A} \xi_{n}-J_{\lambda}^{A} \xi_{m}\right\rangle=0
$$

Let $(\alpha, \beta)$ be a weak cluster point of $\left(J_{\lambda}^{A} \xi_{n}, A_{\lambda} \xi_{n}\right)$. One has

$$
\lim _{n \rightarrow+\infty}\left\langle A_{\lambda} \xi_{n}-\beta, J_{\lambda}^{A} \xi_{n}-\alpha\right\rangle=0 .
$$

By Lemma 2.5, $(\alpha, \beta) \in A$ and $\lambda \beta=F(\xi-\alpha)$. Therefore $\alpha=J_{\lambda}^{A} \xi, \beta=A_{\lambda} \xi$ and $(\alpha, \beta)$ is the unique weak cluster point of $\left(J_{\lambda}^{A} \xi_{n}, A_{\lambda} \xi_{n}\right)$. By Lemma 2.5 one also has

$$
\lim _{n \rightarrow \infty}\left\langle A_{\lambda} \xi_{n}, \xi_{n}-J_{\lambda}^{A} \xi_{n}\right\rangle=\left\langle A_{\lambda} \xi, \xi-J_{\lambda}^{A} \xi\right\rangle
$$

Since $\left\|\xi-J_{\lambda}^{A} \xi\right\|^{2}=\lambda^{2}\left\|A_{\lambda} \xi\right\|^{2}=\left\langle A_{\lambda} \xi, \xi-J_{\lambda}^{A} \xi\right\rangle$, one concludes that $\left(J_{\lambda}^{A} \xi_{n}, A_{\lambda} \xi_{n}\right) \rightarrow$ $(\alpha, \beta)$ in $X \times X^{\prime}$.

For $\xi_{1}, \xi_{2} \in X$, one has

$$
\begin{aligned}
& \lambda\left\langle A_{\lambda} \xi_{1}-A_{\lambda} \xi_{2}, \xi_{1}-\xi_{2}\right\rangle=\left\langle F\left(\xi_{1}-J_{\lambda}^{A} \xi_{1}\right)-F\left(\xi_{2}-J_{\lambda}^{A} \xi_{2}\right), \xi_{1}-\xi_{2}\right\rangle \\
& =\left\langle F\left(\xi_{1}-J_{\lambda}^{A} \xi_{1}\right)-F\left(\xi_{2}-J_{\lambda}^{A} \xi_{2}\right),\left(\xi_{1}-J_{\lambda}^{A} \xi_{1}\right)-\left(\xi_{2}-J_{\lambda}^{A} \xi_{2}\right)\right\rangle \\
& +\left\langle F\left(\xi_{1}-J_{\lambda}^{A} \xi_{1}\right)-F\left(\xi_{2}-J_{\lambda}^{A} \xi_{2}\right), J_{\lambda}^{A} \xi_{1}-J_{\lambda}^{A} \xi_{2}\right\rangle \geq 0
\end{aligned}
$$

thanks to the monotonicity of $A$ and $F$.

Since $A_{\lambda}$ is single-valued and continuous, it is maximal.

Remark 3 . When $X$ is a Hilbert space, $J_{\lambda}^{A}$ is nonexpansive, see [17] and (2.1) in Remark 2. 


\subsection{Convergence of maximal monotone graphs, [10]}

Following Brezis [3] and Attouch [2], the convergence of maximal monotone graphs is defined as follows:

Definition 2.6. Let $A^{n}, A \subset X \times X^{\prime}$ be maximal monotone graphs. The sequence $A^{n}$ converges to $A$ as $n \rightarrow \infty,\left(A^{n} \longmapsto A\right)$, if for every $(\xi, \eta) \in A$ there exists a sequence $\left(\xi_{n}, \eta_{n}\right) \in A^{n}$ such that $\left(\xi_{n}, \eta_{n}\right) \rightarrow(\xi, \eta)$ strongly in $X \times X^{\prime}$ as $n \rightarrow \infty$.

Example 2. If $A^{n}$ and $A$ are everywhere defined, continuous and monotone (see Example 1), and if for every $x \in X, A^{n}(x) \rightarrow A(x)$, then $A^{n} \longmapsto A$.

The converse is true in finite-dimensional spaces

Proposition 2.7. Let $A^{n} \in C\left(\mathbf{R}^{N} ; \mathbf{R}^{N}\right)$ and $A \in C\left(\mathbf{R}^{N} ; \mathbf{R}^{N}\right)$ be monotone. The following are equivalent

(i) $A^{n} \longmapsto A$,

(ii) for every compact set $K \subset \mathbf{R}^{N}, A^{n} \rightarrow A$ uniformly on $K$,

(iii) for every $\xi \in \mathbf{R}^{N}, A^{n}(\xi) \rightarrow A(\xi)$.

Proof. Let us first prove that (i) implies (ii). Suppose $A^{n} \longmapsto A$ and let $K$ be a compact set of $\mathbf{R}^{N}$. Choose $\xi^{i}, i \in\{0, \ldots, N\}$ such that $K$ lies in the interior of the convex hull of $\left(\xi_{n}^{i}\right)$. Since $A^{n} \longmapsto A$, there are sequences $\left(\xi_{n}^{i}\right)_{n \geq 1}$ such that $\left(\xi_{n}^{i}, A^{n}\left(\xi_{n}^{i}\right)\right) \rightarrow\left(\xi^{i}, A\left(\xi^{i}\right)\right)$. In particular, $K$ lies in the interior of the convex hull of $\left(\xi_{n}^{i}\right)$ for large $n$. Let $\left(\xi_{n}\right)$ be a sequence in $K$ such that $\xi_{n} \rightarrow \xi \in K$. By the monotonicity of $A^{n}$,

$$
\limsup _{n \rightarrow \infty}\left\langle A^{n}\left(\xi_{n}\right), \xi_{n}^{i}-\xi_{n}\right\rangle \leq\left\langle A\left(\xi^{i}\right), \xi^{i}-\xi\right\rangle .
$$

Since there is $\delta>0$ such that for large $n, B(0, \delta)$ is contained in the convex hull of the points $\left(\xi_{n}^{i}-\xi_{n}\right)_{0 \leq i \leq N}$, the sequence $\left(A^{n}\left(\xi_{n}\right)\right)_{n \geq 1}$ is bounded. Let $\eta$ be a cluster point of $A^{n}\left(\xi_{n}\right)$. Up to a subsequence, one has $\left\langle A^{n}\left(\xi_{n}\right), \xi_{n}\right\rangle \rightarrow$ $\langle\eta, \xi\rangle$. Since $A$ is continuous, it is maximal, and therefore, by Lemma 2.8 , $\eta=A(\xi)$.

The other implications are trivial.

Remark 4. $(i) \Rightarrow(i i)$ in Proposition 2.7 fails if the dimension of $X$ and $X^{\prime}$ is infinite. Let $X=l^{2}(\mathbf{N})$ and $A^{n}(\xi)=f\left(n\left\langle e_{n}, \xi\right\rangle\right) e_{n}$, where $\left(e_{n}\right)$ is a basis of $l^{2}(\mathbf{N})$ and $f: \mathbf{R} \rightarrow \mathbf{R}$ is nondecreasing continuous, $f(0)=0$ and such that $f(1) \neq 0$. We see that $A^{n}\left(\xi-\left\langle e_{n}, \xi\right\rangle e_{n}\right)=0$ and $\left\langle e_{n}, \xi\right\rangle e_{n} \rightarrow 0$ but (iii) is not true. Taking $\xi=\sum_{m \in \mathbf{N}} \frac{1}{m} e_{m}$, we have the following weak convergence $A^{n}(\xi) \rightarrow 0$, but the strong convergence does not hold.

The convergence of graphs ensures that weak limits of elements of $A^{n}$ are in $A$ provided the duality product of the pairs is preserved at the limit. 
Theorem 2.8. Let $A^{n}, A \subset X \times X^{\prime}$ be maximal monotone graphs, and let $\left(\xi_{n}, \eta_{n}\right) \in A^{n}$ and $(\xi, \eta) \in X \times X^{\prime}$. If, as $n \rightarrow+\infty$,

$$
\begin{aligned}
& A^{n} \longrightarrow A, \\
& \xi_{n} \rightarrow \xi \quad \text { weakly in } X, \\
& \eta_{n} \rightarrow \eta \quad \text { weakly in } X^{\prime}, \\
& \liminf _{n \rightarrow+\infty}\left\langle\eta_{n}, \xi_{n}\right\rangle \leq\langle\eta, \xi\rangle,
\end{aligned}
$$

then $(\xi, \eta) \in A$ and

$$
\liminf _{n \rightarrow+\infty}\left\langle\eta_{n}, \xi_{n}\right\rangle=\langle\eta, \xi\rangle
$$

Remark 5. If $\left(\xi_{n}, \eta_{n}\right) \rightarrow(\xi, \eta)$ strongly in $X \times X^{\prime}$, then (2.4) is satisfied, i.e. every strongly converging sequence of elements of $A^{n}$ is in $A$. The same holds when only $\left(\xi_{n}\right)$ (resp. $\left(\eta_{n}\right)$ ) converges strongly in $X$ (resp. in $X^{\prime}$ ).

The proof of Theorem 2.8 is similar to that of Lemma 2.5.

Theorem 2.9. Let $A^{n}, A \subset X \times X^{\prime}$ be maximal monotone graphs and $\lambda>0$. The following are equivalent:

(i) $A^{n} \longmapsto A$, as $n \rightarrow \infty$,

(ii) $\forall \zeta \in X, J_{\lambda}^{A^{n}} \zeta \rightarrow J_{\lambda}^{A} \zeta$ as $n \rightarrow \infty$

(iii) $\forall \zeta \in X, A_{\lambda}^{n} \zeta \rightarrow A_{\lambda} \zeta$ as $n \rightarrow \infty$,

(iv) $A_{\lambda}^{n} \longmapsto A_{\lambda}$, as $n \rightarrow \infty$.

Moreover, the convergences $J_{\lambda}^{A^{n}} \zeta \rightarrow J_{\lambda}^{A} \zeta$ and $A_{\lambda}^{n} \zeta \rightarrow A_{\lambda} \zeta$ are uniform on strongly compact sets of $X$.

Remark 6 . Note that $\lambda$ does not play any role in (i). Therefore if there exists $\lambda_{0}>0$ such that any of (ii)-(iv) holds, then (ii)-(iv) hold for every $\lambda>0$.

Proof. Let us prove that (i) implies that $J_{\lambda}^{A^{n}} \zeta \rightarrow J_{\lambda}^{A} \zeta$ and $A_{\lambda}^{n} \zeta \rightarrow A_{\lambda} \zeta$ uniformly on compact subsets of $X$. This will imply (ii) and (iii). Let $\left(\zeta_{n}\right)_{n \geq 1} \in X$ be such that $\zeta_{n} \rightarrow \zeta$ strongly in $X$. Define $(\xi, \eta)=\left(J_{\lambda}^{A} \zeta, A_{\lambda} \zeta\right)$. Since $A^{n} \longmapsto A$ as $n \rightarrow \infty$, there is a sequence $\left(\xi_{n}, \eta_{n}\right) \in A^{n}$ such that $\left(\xi_{n}, \eta_{n}\right) \rightarrow(\xi, \eta)$ strongly in $X \times X^{\prime}$. By the monotonicity of $A^{n}$,

$$
\begin{aligned}
& \lambda\left\|A_{\lambda}^{n} \zeta_{n}\right\|^{2}=\left\langle A_{\lambda}^{n} \zeta_{n}, \zeta_{n}-J_{\lambda}^{A^{n}} \zeta_{n}\right\rangle \\
& \quad \leq\left\langle\eta_{n}, \xi_{n}-J_{\lambda}^{A^{n}} \zeta_{n}\right\rangle+\left\langle A_{\lambda}^{n} \zeta_{n}, \zeta_{n}-\xi_{n}\right\rangle \leq\left\|A_{\lambda}^{n} \zeta_{n}\right\|\left(\lambda\left\|\eta_{n}\right\|+\left\|\zeta_{n}-\xi_{n}\right\|\right) .
\end{aligned}
$$

Hence the sequences $A_{\lambda}^{n} \zeta_{n}$ and $J_{\lambda}^{A^{n}} \zeta_{n}$ are bounded in $X$ and $X^{\prime}$. Let $(\alpha, \beta) \in$ $X \times X^{\prime}$ be a weak cluster point of the sequence $\left(J_{\lambda}^{A^{n}} \zeta_{n}, A_{\lambda}^{n} \zeta_{n}\right)$, i.e., up to a 
subsequence, $\left(J_{\lambda}^{A^{n}} \zeta_{n}, A_{\lambda}^{n} \zeta_{n}\right) \rightarrow(\alpha, \beta)$ weakly in $X \times X^{\prime}$. By the monotonicity of $F$,

$$
\left\langle A_{\lambda}^{n} \zeta_{n}-A_{\lambda}^{m} \zeta_{m},\left(\zeta_{n}-J_{\lambda}^{A^{n}} \zeta_{n}\right)-\left(\zeta_{m}-J_{\lambda}^{A^{m}} \zeta_{m}\right)\right\rangle \geq 0
$$

Letting $m \rightarrow \infty$ gives

$$
\begin{aligned}
\left\langle A_{\lambda}^{n} \zeta_{n}, J_{\lambda}^{A^{n}} \zeta_{n}\right\rangle+\limsup _{m \rightarrow+\infty}\left\langle A_{\lambda}^{m} \zeta_{m}, J_{\lambda}^{A^{m}} \zeta_{m}\right\rangle \leq & \left\langle A_{\lambda}^{n} \zeta_{n}, \zeta_{n}-\zeta+\alpha\right\rangle \\
& -\left\langle\beta, \zeta_{n}-\zeta-J_{\lambda}^{A^{n}} \zeta_{n}\right\rangle .
\end{aligned}
$$

Letting now $n \rightarrow \infty$, we obtain

$$
\limsup _{n \rightarrow+\infty}\left\langle A_{\lambda}^{n} \zeta_{n}, J_{\lambda}^{A^{n}} \zeta_{n}\right\rangle \leq\langle\beta, \alpha\rangle .
$$

By Theorem 2.8, $(\alpha, \beta) \in A$, and $\liminf _{n \rightarrow \infty}\left\langle A_{\lambda}^{n} \zeta_{n}, J_{\lambda}^{A^{n}} \zeta_{n}\right\rangle \geq\langle\beta, \alpha\rangle$. Hence,

$$
\begin{aligned}
& \limsup _{n \rightarrow \infty}\left\|A_{\lambda}^{n} \zeta_{n}\right\|\left\|\zeta_{n}-J_{\lambda}^{A^{n}} \zeta\right\|= \\
& \qquad \limsup _{n \rightarrow \infty}\left\langle A_{\lambda}^{n} \zeta_{n}, \zeta_{n}-J_{\lambda}^{A^{n}} \zeta_{n}\right\rangle \leq\langle\beta, \zeta-\alpha\rangle \leq\|\beta\|\|\zeta-\alpha\| .
\end{aligned}
$$

Therefore $\left(\zeta-J_{\lambda}^{A^{n}} \zeta_{n}, A_{\lambda}^{n} \zeta_{n}\right) \rightarrow(\zeta-\alpha, \beta)$ strongly in $X \times X^{\prime}$. By the continuity of $F, \lambda F(\beta)=\zeta-\alpha$, and therefore $(\alpha, \beta)=\left(J_{\lambda}^{A} \zeta, A_{\lambda} \zeta\right)$. One concludes that $\left(J_{\lambda}^{A^{n}} \zeta_{n}, A_{\lambda}^{n} \zeta_{n}\right) \rightarrow\left(J_{\lambda}^{A} \zeta, A_{\lambda} \zeta\right)$ in $X \times X^{\prime}$ as $n \rightarrow \infty$.

The equivalence between (ii) and (iii) comes from the continuity of the duality mapping $F$.

Assertion (iii) implies (iv) (see Example 2).

Finally, (iv) implies (i): Let $(\xi, \eta) \in A$, and define $\zeta=\xi+F^{-1}(\lambda \eta)$. By the assumption, there is a sequence $\zeta_{n} \in X$ such that $\left(\zeta_{n}, A_{\lambda}^{n} \zeta_{n}\right) \rightarrow$ $\left(\zeta, A_{\lambda} \zeta\right)$ in $X \times X^{\prime}$. By the continuity of $F^{-1}, J_{\lambda}^{A_{n}} \zeta_{n}=\zeta_{n}-F^{-1}\left(\lambda A_{\lambda}^{n} \zeta_{n}\right) \rightarrow$ $\zeta-F^{-1}\left(\lambda A_{\lambda} \zeta\right)=J_{\lambda}^{A} \zeta$. Therefore, $\left(J_{\lambda}^{A^{n}} \zeta_{n}, A_{\lambda}^{n} \zeta_{n}\right) \rightarrow\left(J_{\lambda}^{A} \zeta, A_{\lambda} \zeta\right)=(\xi, \eta)$ strongly in $X \times X^{\prime}$.

Remark 7. If $X$ is a Hilbert space, $\phi_{A^{n}}: X \rightarrow X$ and $\phi_{A}: X \rightarrow X$ are the nonexpansive functions associated to $A^{n}$ and $A$ in Remark 2, then the propositions of the Theorem are equivalent with:

For every $\xi \in X, \phi_{A^{n}}(\xi) \rightarrow \phi_{A}(\xi)$ as $n \rightarrow \infty$.

\subsection{Graph valued measurable functions}

From now on, we shall assume that $X$, and hence $X^{\prime}$, is separable. The set of maximal monotone operators from $X$ to $X^{\prime}$ is denoted by $\mathfrak{M}\left(X \times X^{\prime}\right)$. In this section $(\Omega, \mathcal{T}, \mu)$ will be a $\sigma$-finite $\mu$-complete measure-space. The characteristic function associated to the set $A$, is denoted by $\chi_{A}$.

The following definition can be found in [6]. 
Definition 2.10. A function $A: \Omega \rightarrow \mathfrak{M}\left(X \times X^{\prime}\right)$ is measurable if and only if for every open set $U \subset X \times X^{\prime}$ (resp closed set, Borel set, open ball, closed ball),

$$
\{t \in \Omega \mid A(t) \cap U \neq \emptyset\}
$$

is measurable in $\Omega$.

The following proposition was proved in [1] for the Hilbert case, using results of [6].

Proposition 2.11. Let $A: \Omega \rightarrow \mathfrak{M}\left(X \times X^{\prime}\right)$, let $\lambda>0$ and let $E$ be dense in $X$. The following are equivalent:

(i) A is measurable,

(ii) for every $\zeta \in E, t \mapsto J_{\lambda}^{A(t)} \zeta$ is measurable,

(iii) for every $\zeta \in E, t \mapsto A(t)_{\lambda} \zeta$ is measurable.

Remark 8 . Note that neither $\lambda$ nor $E$ play any role in (i). Therefore if there exists $\lambda_{0}>0$ and a dense set $E$ such that either of (ii) or (iii) holds, then (ii) and (iii) hold for every $\lambda>0$ and for every $\zeta \in X$.

Proof. Without loss of generality, set $\lambda=1$.

Assume (i). Let $G \subset X$ be closed. For every $\zeta \in X$, set

$$
C_{\zeta}=\left\{(\xi, \eta) \in G \times X^{\prime} \mid \xi+F^{-1}(\eta)=\zeta\right\} .
$$

From the continuity of $F^{-1}$, it follows that $C_{\zeta}$ is closed. Now we have

$$
\left\{t \in \Omega \mid J_{1}^{A(t)} \zeta \in G\right\}=\left\{t \in \Omega \mid A(t) \cap C_{\zeta} \neq \emptyset\right\},
$$

the latter is measurable by (i), so that (ii) is satisfied.

To show that (iii) implies (i), we can assume that $E$ is countable. For $(\xi, \eta) \in A(t)$, setting $\zeta=\xi+F^{-1}(\eta)$ gives $\xi=J_{1}^{A(t)} \zeta, \eta=A(t)_{1} \zeta$, therefore by the continuity of $J_{1}^{A(t)} \zeta$ and of $A(t)_{1} \zeta$ with respect to $\zeta$ and the density of $E$, one has

$$
\begin{aligned}
& \{t \in \Omega \mid A(t) \cap U \neq \emptyset\} \\
& =\bigcup_{\zeta \in X}\left\{t \in \Omega \mid\left(J_{1}^{A(t)} \zeta, A(t){ }_{1} \zeta\right) \in U\right\} \\
& \quad=\bigcup_{\zeta \in E}\left\{t \in \Omega \mid\left(J_{1}^{A(t)} \zeta, A(t)_{1} \zeta\right) \in U\right\}
\end{aligned}
$$

thus, the left-hand side is measurable.

Remark 9. If $X$ is a Hilbert space, then $A: \Omega \rightarrow \mathfrak{M}\left(X \times X^{\prime}\right)$ is measurable if and only if the associated function $t \rightarrow \phi_{A(t)}(\zeta)$ (by Remark 2) is measurable for every $\zeta \in X$ (see Remark 2.2. in [13]). 


\subsection{Canonical extensions of maximal monotone graphs}

Given a function $A: \Omega \rightarrow \mathfrak{M}\left(X \times X^{\prime}\right)$, one can define a monotone graph from $L^{p}(\Omega ; X)$ to $L^{q}\left(\Omega ; X^{\prime}\right)$, where $1 / p+1 / q=1$, as follows:

Definition 2.12. Let $A: \Omega \rightarrow \mathfrak{M}\left(X \times X^{\prime}\right)$, the canonical extension of $A$ from $L^{p}(\Omega ; X)$ to $L^{q}\left(\Omega ; X^{\prime}\right)$, where $1 / p+1 / q=1$, is defined by:

$$
\mathcal{A}=\left\{(u, v) \in L^{p}(\Omega ; X) \times L^{q}\left(\Omega ; X^{\prime}\right) \mid(u(t), v(t)) \in A(t) \text { for a.e. } t \in \Omega\right\} .
$$

One readily checks that $\mathcal{A}$ is monotone.

Proposition 2.13. Let $A: \Omega \rightarrow \mathfrak{M}\left(X \times X^{\prime}\right)$ be measurable. If $\mathcal{A} \neq \emptyset$, then $\mathcal{A}$ is maximal monotone.

Remark 10. The maximality of $A(t)$ for almost every $t \in \Omega$ is not sufficient in order to ensure the maximality of $\mathcal{A}$ as the latter could be empty: $\Omega=(0,1)$ and $A(t)=\left\{(\xi, \eta) \in \mathbf{R}^{N} \times \mathbf{R}^{N}: \eta=t^{-1 / q}\right\}$.

Proof. Since $\mathcal{A} \neq \emptyset$, there exists $(\alpha, \beta) \in \mathcal{A}$. Suppose $(u, v) \in L^{p}(\Omega ; X) \times$ $L^{q}\left(\Omega ; X^{\prime}\right)$ is such that for every $\left(u^{\prime}, v^{\prime}\right) \in \mathcal{A}$,

$$
\int_{\Omega}\left\langle v^{\prime}(t)-v(t), u^{\prime}(t)-u(t)\right\rangle \mathrm{d} \mu \geq 0 .
$$

For every $t \in \Omega$, set

$$
\begin{aligned}
& u_{*}(t)=J_{1}^{A(t)}\left(u(t)+F^{-1}(v(t))\right), \\
& v_{*}(t)=A(t)_{1}\left(u(t)+F^{-1}(v(t))\right) .
\end{aligned}
$$

By construction, $\left(u_{*}(t), v_{*}(t)\right) \in A(t)$ for almost every $t \in \Omega$. For $k>0$, define

$$
\Omega_{k}=\left\{t \in \Omega \mid\left\|u_{*}(t)\right\| \leq k\|u(t)\| \text { and }\left\|v_{*}(t)\right\| \leq k\|v(t)\|\right\}
$$

and

$$
\left(u_{k}, v_{k}\right)=\chi_{\Omega_{k}}\left(u_{*}, v_{*}\right)+\chi_{\Omega \backslash \Omega_{k}}(\alpha, \beta) .
$$

One has $\left(u_{k}, v_{k}\right) \in \mathcal{A}$, so that

$$
\int_{\Omega}\left\langle v_{k}(t)-v(t), u_{k}(t)-u(t)\right\rangle \mathrm{d} \mu \geq 0
$$

which reads

$$
\int_{\Omega_{k}}\left\langle v_{*}(t)-v(t), u_{*}(t)-u(t)\right\rangle \mathrm{d} \mu \geq-\int_{\Omega \backslash \Omega_{k}}\langle\beta(t)-v(t), \alpha(t)-u(t)\rangle \mathrm{d} \mu
$$


Since $u_{*}(t)+F^{-1}\left(v_{*}(t)\right)=u(t)+F^{-1}(v(t))$, we have for a.e. $t \in \Omega$, by the monotonicity of $F$,

$$
\left\langle v(t)-v_{*}(t), u(t)-u_{*}(t)\right\rangle=\left\langle v(t)-v_{*}(t), F^{-1}\left(v_{*}(t)\right)-F^{-1}(v(t))\right\rangle \leq 0,
$$

and

$$
\begin{aligned}
0 \geq \int_{\Omega_{k}}\left\langle v(t)-v_{*}(t), F^{-1}\left(v_{*}(t)\right)\right. & \left.-F^{-1}(v(t))\right\rangle \mathrm{d} \mu \\
& \geq-\int_{\Omega \backslash \Omega_{k}}\langle\beta(t)-v(t), \alpha(t)-u(t)\rangle \mathrm{d} \mu .
\end{aligned}
$$

Lebesgue's monotone convergence theorem now gives

$$
0=\int_{\Omega}\left\langle v(t)-v_{*}(t), F^{-1}\left(v_{*}(t)\right)-F^{-1}(v(t))\right\rangle \mathrm{d} \mu
$$

so that by definition of $F^{-1}$

$$
\begin{array}{r}
0=\int_{\Omega}\left(-\left\|v_{*}(t)\right\|^{2}-\|v(t)\|^{2}+\left\langle v_{*}(t), F^{-1}(v(t))\right\rangle+\left\langle v(t), F^{-1}\left(v_{*}(t)\right)\right\rangle\right) \mathrm{d} \mu \\
\leq \int_{\Omega}-\left(\|v(t)\|-\left\|v_{*}(t)\right\|\right)^{2} \mathrm{~d} \mu .
\end{array}
$$

This implies $\|v(t)\|=\left\|v_{*}(t)\right\|$ for almost every $t \in \Omega$ and $\left\langle v_{*}(t), F^{-1}(v(t))\right\rangle=$ $\|v(t)\|\left\|v_{*}(t)\right\|$. Therefore, $(u, v)=\left(u_{*}, v_{*}\right)$. Since $(u, v) \in L^{p}(\Omega ; X) \times$ $L^{q}\left(\Omega ; X^{\prime}\right)$ and $\left(u_{*}(t), v_{*}(t)\right) \in A(t)$, for almost every $t$, one concludes that $(u, v)$ belongs $\mathcal{A}$.

A particular important class of maximal graphs is defined by

Definition 2.14. For $m$ measurable from $\Omega$ to $\overline{\mathbf{R}}, \alpha \in \mathbf{R}$ and $p>1$, $\mathfrak{M}(\Omega, X, p, \alpha, m)$ is the set of $A: \Omega \rightarrow \mathfrak{M}\left(X \times X^{\prime}\right)$ such that $A$ is measurable and such that for almost every $t \in \Omega$, for every $(\xi, \eta) \in A(t)$,

$$
\alpha\left(\frac{\|\xi\|^{p}}{p}+\frac{\|\eta\|^{q}}{q}\right) \leq\langle\eta, \xi\rangle+m(t)
$$

where $p^{-1}+q^{-1}=1$.

Remark 11. If $\alpha>1$, then $\mathfrak{M}(\Omega, X, p, \alpha, m)$ is empty: By Young's inequality, the graph $A(t)$ should be bounded in $X \times X^{\prime}$ for almost every $t \in \Omega$, in contradiction with the maximality of $A(t)$.

Remark 12. If $-1 \leq \alpha \leq 1$, then $\mathfrak{M}(\Omega, X, p, \alpha, m)$ is not empty if and only if $m \geq 0$. The graph-valued function $A(t)=\left\{\left(\xi,\|\xi\|^{p-2} F(\xi)\right) \mid \xi \in X\right\}$ is in $\mathfrak{M}(\Omega, X, p, \alpha, m)$ for every $\alpha \leq 1$ and $m \geq 0$. Conversely, suppose $A \in$ $\mathfrak{M}(\Omega, X, p, \alpha, m)$. Since $A(t)$ is maximal almost everywhere, by Theorem 2.18 (see below), there is $(\xi, \eta) \in A(t)$ such that $\eta=-\|\xi\|^{p-2} F(\xi)$. By (2.6), $m(t) \geq 0$. 
Remark 13. By Young's inequality, if $\alpha \leq-1$ and $m \geq 0$, then $\mathfrak{M}(\Omega, X, p, \alpha, m)$ is the set of measurable graph-valued functions. The condition $m \geq 0$ is necessary: If $m(t)<0$, then $(0,0) \notin A(t)$, which means that in this case, any graph which contains $(0,0)$ is not in $\mathfrak{M}(\Omega, X, p, \alpha, m)$.

Remark 14. If $\alpha<-1$ and $X \neq\{0\}$, then $\mathfrak{M}(\Omega, X, p, \alpha, m)$ is never empty since it always contains the graph-valued function $A(t)=\{(\xi, \bar{\eta}(t)) \mid \xi \in X\}$, where $\bar{\eta}(t) \in X^{\prime}$ is such that $-(\alpha+1)\|\bar{\eta}(t)\|^{q} / q \geq m(t)$.

Corollary 2.15. Let $\alpha>0$ and $m \in L^{1}(\Omega)$. If $A \in \mathfrak{M}(\Omega, X, p, \alpha, m)$, then $\mathcal{A}$ is maximal monotone.

Moreover, $D(\mathcal{A})=L^{p}(\Omega ; X)$ and $\mathcal{A}$ is surjective.

Remark 15. For the maximality of $\mathcal{A}, \alpha>0$ can be weakened to $\alpha>-1$ (Proposition 2.20 below). The condition $\alpha>0$ is optimal for $\mathcal{A}$ to be everywhere defined and onto. Indeed, let $A(t)=\{(0, \eta) \mid \eta \in X\}$. Condition 2.6 is satisfied with $m=0$ and $\alpha=0$, and $D(\mathcal{A})=\{0\}$. Similarly, with $A(t)=\{(\xi, 0) \mid \xi \in X\}, \mathcal{A}$ is not surjective.

Proof. Let $u \in L^{p}(\Omega ; X)$ and let for $\lambda>0$,

$$
\left(u_{\lambda}(t), v_{\lambda}(t)\right)=\left(J_{\lambda}^{A(t)} u(t), A(t)_{\lambda} v(t)\right) .
$$

By definition, $\left(u_{\lambda}(t), v_{\lambda}(t)\right) \in A(t)$ for almost every $t \in \Omega$. By (2.6), one has

$$
\begin{aligned}
& \alpha\left(\frac{\left\|u_{\lambda}(t)\right\|^{p}}{p}+\frac{\left\|v_{\lambda}(t)\right\|^{q}}{q}\right) \\
& \quad \leq\left\langle v_{\lambda}(t), u_{\lambda}(t)\right\rangle+m(t)=-\lambda\left\|v_{\lambda}\right\|^{2}+\left\langle v_{\lambda}(t), u(t)\right\rangle+m(t) \\
& \quad \leq\left\langle v_{\lambda}(t), u(t)\right\rangle+m(t) .
\end{aligned}
$$

Hence, there is $C>0$ such that for almost every $t \in \Omega$,

$$
\frac{\alpha}{2}\left(\frac{\left\|u_{\lambda}(t)\right\|^{p}}{p}+\frac{\left\|v_{\lambda}(t)\right\|^{q}}{q}\right) \leq C\|u(t)\|^{p}+m(t) .
$$

In particular, $\left(u_{\lambda}, v_{\lambda}\right) \in L^{p}(\Omega ; X) \times L^{q}\left(\Omega ; X^{\prime}\right)$. This $\mathcal{A}$ is not empty hence maximal by Proposition 2.13.

Integrating (2.7), gives

$$
\frac{\alpha}{2 q} \int_{\Omega}\left\|v_{\lambda}\right\|^{q} \mathrm{~d} \mu \leq C \int_{\Omega}\|u\|^{p} d \mu+\int_{\Omega} m \mathrm{~d} \mu
$$

Therefore

$$
\lim _{\lambda \rightarrow 0} \int_{\Omega}\left\|\lambda v_{\lambda}\right\|^{q} \mathrm{~d} \mu=0
$$

and up to a subsequence, for almost every $t \in \Omega$,

$$
u_{\lambda}(t)-u(t)=\lambda F^{-1}\left(v_{\lambda}(t)\right) \rightarrow 0,
$$


as $\lambda \rightarrow 0$. Together with (2.7), Lebesgue's dominated convergence Theorem implies $u_{\lambda} \rightarrow u$ in $L^{p}(\Omega ; X)$. Since the sequence $v_{\lambda}$ is bounded in $L^{q}\left(\Omega ; X^{\prime}\right)$, it is weakly compact, and there is $v \in L^{q}\left(\Omega ; X^{\prime}\right)$ such that $v_{\lambda} \rightarrow v$ weakly in $L^{q}\left(\Omega ; X^{\prime}\right)$. Since $\mathcal{A}$ is maximal, Lemma 2.5 is applicable, and $(u, v) \in \mathcal{A}$.

Note that $\mathcal{A}^{-1}=\{(v, u) \mid(u, v) \in \mathcal{A}\}$ satisfies the same assumptions as $\mathcal{A}$ (with $p$ and $q$ reversed). By the proof above, $D\left(\mathcal{A}^{-1}\right)=X^{\prime}$, which is equivalent with the surjectivity of $\mathcal{A}$.

\subsection{Convergence of canonical extensions}

Given functions $A, A^{n}: \Omega \rightarrow \mathfrak{M}\left(X \times X^{\prime}\right)$ and their canonical extensions $\mathcal{A}, \mathcal{A}^{n}$ (by (2.5)), we consider the question whether the pointwise convergence $A^{n}(t) \longmapsto A(t)$ implies the convergence of the induced graphs $\mathcal{A}^{n} \longmapsto \mathcal{A}$.

Theorem 2.16. Let $A, A^{n}: \Omega \rightarrow \mathfrak{M}\left(X \times X^{\prime}\right)$ be measurable. Assume

(i) for almost every $t \in \Omega, A^{n}(t) \longmapsto A(t)$ as $n \rightarrow \infty$,

(ii) $\mathcal{A}$ and $\mathcal{A}^{n}$ are maximal monotone,

(iii) there exists $\left(\alpha_{n}, \beta_{n}\right) \in \mathcal{A}^{n}$ and $(\alpha, \beta) \in L^{p}(\Omega ; X) \times L^{q}\left(\Omega ; X^{\prime}\right)$ such that $\left(\alpha_{n}, \beta_{n}\right) \rightarrow(\alpha, \beta)$ strongly in $L^{p}(\Omega ; X) \times L^{q}\left(\Omega ; X^{\prime}\right)$ as $n \rightarrow \infty$,

then $\mathcal{A}^{n} \longmapsto \mathcal{A}$.

Remark 16. Assumption (iii) cannot be dropped. One can have $A^{n}(t) \longmapsto$ $A(t)$ for every $t \in \Omega$ while $\mathcal{A}^{n} \nrightarrow \mathcal{A}$. For example, let $X=\mathbf{R}$, and let $\Omega=(0,1)$. Define $A^{n}(t)=\left\{(x, y) \mid y=n^{1 / q} \chi_{(0,1 / n)}(t)\right\}$ and $A(t)=$ $\{(x, y) \mid y=0\}$. One has $A^{n}(t) \longmapsto A(t)$ for every $t \in \Omega$. On the other hand for every $\left(u_{n}, v_{n}\right) \in \mathcal{A}^{n}$ and $(u, v) \in \mathcal{A}$, one has $\left\|v_{n}\right\|_{L^{q}}=1$ and $\|v\|_{L^{q}}=0$ so that $v_{n}$ can not converge to $v$ in $L^{q}(\Omega)$ as $n \rightarrow \infty$, whence $\mathcal{A}^{n} \longrightarrow \mathcal{A}$ is impossible.

Proof. Let $(u, v) \in \mathcal{A}$. For $t \in \Omega$, let $w(t)=u(t)+F^{-1}(v(t))$, so that $(u(t), v(t))=\left(J_{1}^{A(t)}(w(t)), A(t)_{1}(w(t))\right.$. Define

$$
\Omega^{\prime}=\left\{t \in \Omega \mid\left\|J_{1}^{A^{n}(t)} w(t)\right\| \leq 2\|u(t)\| \text { and }\left\|A^{n}(t){ }_{1} w(t)\right\| \leq 2\|v(t)\|\right\},
$$

and, for $n \geq 1$,

$$
\left(u_{n}(t), v_{n}(t)\right)= \begin{cases}\left(J_{1}^{A^{n}(t)}(w(t)), A^{n}(t)_{1}(w(t))\right) & \text { if } t \in \Omega^{\prime}, \\ \left(\alpha_{n}(t), \beta_{n}(t)\right) & \text { if } t \notin \Omega^{\prime}\end{cases}
$$

By construction, $\left(u_{n}, v_{n}\right)$ is in $\mathcal{A}^{n}$. By dominated convergence, $\left(u_{n}, v_{n}\right) \rightarrow$ $(u, v)$ strongly in $L^{p}(\Omega ; X) \times L^{q}\left(\Omega ; X^{\prime}\right)$. 
Corollary 2.17. Assume $\alpha>0, m_{n} \in L^{1}(\Omega), A^{n} \in \mathfrak{M}\left(\Omega, X, p, \alpha, m_{n}\right)$ and $A: \Omega \rightarrow \mathfrak{M}\left(X \times X^{\prime}\right)$ measurable. If $m_{n}$ converges strongly to $m$ in $L^{1}(\Omega)$ and if for almost every $t$ in $\Omega, A^{n}(t) \longmapsto A(t)$ holds, then $A \in \mathfrak{M}(\Omega, X, p, \alpha, m)$ and $\mathcal{A}^{n} \longmapsto \mathcal{A}$.

Proof. We apply Theorem 2.16. Hypothesis $(i)$ is satisfied. By Corollary $2.15, \mathcal{A}^{n}$ is maximal monotone. Up to a subsequence (not relabeled), $m_{n}$ converge to $m$ a.e. and remains dominated in $L^{1}(\Omega)$. Hence, $A^{n}(t) \longmapsto A(t)$, for a.e. $t \in \Omega$ implies that $A \in \mathfrak{M}(\Omega, X, p, \alpha, m)$ so $\mathcal{A}$ is maximal.

We now check hypothesis $($ iii). Define

$$
\left(u_{n}(t), v_{n}(t)\right)=\left(J_{1}^{A^{n}(t)} 0, A^{n}(t)_{1} 0\right) .
$$

Clearly, $\left(u_{n}(t), v_{n}(t)\right) \in A^{n}(t)$ for a.e. $t \in \Omega$ and $\left\langle v_{n}(t), u_{n}(t)\right\rangle=-\left\|u_{n}(t)\right\|^{2} \leq$ 0 . Combined with (2.6), this yields

$$
\alpha\left(\frac{\left\|u_{n}(t)\right\|^{p}}{p}+\frac{\left\|v_{n}(t)\right\|^{q}}{q}\right) \leq m_{n}(t) .
$$

Therefore, $\left(u_{n}, v_{n}\right) \in L^{p}(\Omega ; X) \times L^{q}\left(\Omega ; X^{\prime}\right)$ for every $n \geq 0$ and $\left(u_{n}, v_{n}\right) \in$ $\mathcal{A}^{n}$. By Theorem 2.9 applied, for almost every $t \in \Omega$, to $A^{n}(t)$,

$$
\left(u_{n}(t), v_{n}(t)\right) \rightarrow(u(t), v(t)) \text {, strongly in } X \times X^{\prime},
$$

where $(u(t), v(t))=\left(J_{1}^{A(t)} 0, A(t)_{1} 0\right)$. Now Lebesgue's dominated convergence Theorem implies that for any sequenc $n_{k} \rightarrow \infty$, there is a subsequence $n_{k_{j}}$ such that

$$
\left(u_{n_{k_{j}}}, v_{n_{k_{j}}}\right) \rightarrow(u, v) \text { strongly in } L^{p}(\Omega ; X) \times L^{q}\left(\Omega ; X^{\prime}\right) .
$$

The argument above implies the convergence of the whole sequence $\left(u_{n}, v_{n}\right)$ to $(u, v)$.

\subsection{Changing the duality mapping}

Theorem 2.2 is a particular case of a more general result [5]: $F$ is a monotone continuous coercive map, and there is uniqueness when $F$ is strictly monotone, i.e. $\left\langle F\left(\xi_{1}\right)-F\left(\xi_{2}\right), \xi_{1}-\xi_{2}\right\rangle>0$ when $\xi_{1} \neq \xi_{2} \in X$. In view of the proof of Proposition 4.2, we consider $F_{p}$ defined for $\xi \in X$ by

$$
F_{p}(\xi)=F(\xi)\|\xi\|^{p-2}, \forall \xi \in X
$$

It satisfies

$$
\left\langle F_{p}(\xi), \xi\right\rangle=\|\xi\|^{p}=\left\|F_{p}(\xi)\right\|^{q} .
$$

The case of Theorem 2.2 corresponds to $p=2$. Browder's results in this case are 
Proposition 2.18 (Browder [4]). Let $A \subset X \times X^{\prime}$ be a monotone graph. The graph $A$ is maximal monotone if and only if $A+F_{p}$ is a surjective map, i.e. for every $\eta \in X^{\prime}$, there is $\xi \in X$ such that $\left(\xi, \eta-F_{p}(\xi)\right) \in A$.

Similarly to the case $p=2$, the following definition makes sense:

Definition 2.19. Let $A \subset X \times X^{\prime}$ be a maximal monotone graph. For every $\xi \in X$ and $\lambda>0,\left(J_{\lambda, p}^{A} \xi, A_{\lambda, p} \xi\right)$ denotes the unique pair in $A$ such that

$$
F_{p}\left(\xi-J_{\lambda, p}^{A} \xi\right)=\lambda A_{\lambda, p} \xi
$$

With the previous definition and Theorem 2.18, all the results of section 2 in the case of the $F_{p}$ duality mapping hold with obvious modifications in the proofs.

The use of $F_{p}$ yields improvements of Corollaries 2.15 and 2.17:

Proposition 2.20. The first parts of Corollary 2.15, and Corollary 2.17 are valid under the hypothesis $\alpha>-1$ (instead of $\alpha>0$ ).

Proof. This is based on the fact that if

$$
(u(t), v(t))=\left(J_{1, p}^{A(t)} 0, A(t)_{1, p} 0\right),
$$

one has $v(t)=-F_{p}(u(t))$, and under hypothesis (2.6), this gives

$$
\frac{\|u(t)\|^{p}}{p}+\frac{\|v(t)\|^{q}}{q}=-\langle v(t), u(t)\rangle \leq m(t)-\alpha\left(\frac{\|u(t)\|^{p}}{p}+\frac{\|v(t)\|^{q}}{q}\right) .
$$

Remark 17. By Young's inequality, every graph $A$ satisfies (2.6) with $\alpha=-1$. Together with Remarks 10 and 16 this means that the condition $\alpha>-1$ is optimal.

\section{The periodic unfolding}

The periodic unfolding operator was introduced by Cioranescu, Damlamian and Griso [9]. We recall the definitions and properties of this operator. The proofs can be found in $[9,11,16]$.

\subsection{Definition of the periodic unfolding operator and prop- erties}

In $\mathbf{R}^{N}$, let $Y$ be a reference cell (e.g. $] 0,1\left[{ }^{N}\right.$, or more generally a set having the paving property with respect to a basis $\left(b_{1}, \ldots, b_{N}\right)$ defining the periods).

For $y \in \mathbf{R}^{N},[y]_{Y}$ denotes the unique integer combination $\sum_{j=1}^{N} k_{j} b_{j}$, with $k_{j} \in \mathbf{Z}$, of the periods such that $y-[y]_{Y}$ belongs to $Y$ and define

$$
\{y\}_{Y}=y-[y]_{Y} \in Y \text {. }
$$


Definition 3.1. Let $Y$ be a reference cell, $\epsilon$ a positive number, $S$ a set and a map $u: \mathbf{R}^{N} \rightarrow S$. The unfolding operator $\mathcal{T}_{\epsilon}^{Y}$ is defined by

$$
\begin{aligned}
\mathcal{T}_{\epsilon}^{Y}(u): \mathbf{R}^{N} \times \mathbf{R}^{N} & \rightarrow S \\
(x, y) & \mapsto \mathcal{T}_{\epsilon}^{Y}(u)(x, y)=u\left(\epsilon\left[\frac{x}{\epsilon}\right]_{Y}+\epsilon y\right) .
\end{aligned}
$$

One readily sees that for every $x \in \mathbf{R}^{N}$,

$$
\mathcal{T}_{\epsilon}^{Y}(u)(x,\{x / \epsilon\})=u(x) .
$$

Moreover, $\mathcal{T}_{\epsilon}^{Y}(u)$ is invariant under the following action of $\mathbf{Z}^{N}$ : for $k \in \mathbf{Z}^{N}$,

$$
\mathcal{T}_{\epsilon}^{Y}(u)(x+\epsilon k, y-k)=\mathcal{T}_{\epsilon}^{Y}(u)(x, y) .
$$

If $u: \mathbf{R}^{N} \rightarrow S$ and $f: S \rightarrow S^{\prime}$, then

$$
\mathcal{T}_{\epsilon}^{Y}(f \circ u)=f \circ \mathcal{T}_{\epsilon}^{Y}(u)
$$

In particular if $u: \mathbf{R}^{N} \rightarrow S$ and $v: \mathbf{R}^{N} \rightarrow T$, the preceding property applied to the projections $P:(u, v) \mapsto u$ and $Q:(u, v) \mapsto v$ yields

$$
\mathcal{T}_{\epsilon}^{Y}((u, v))=\left(\mathcal{T}_{\epsilon}^{Y}(u), \mathcal{T}_{\epsilon}^{Y}(v)\right) .
$$

Therefore, if $F: S \times T \rightarrow R$,

$$
\mathcal{T}_{\epsilon}^{Y}(F(u, v))=F\left(\mathcal{T}_{\epsilon}^{Y}(u), \mathcal{T}_{\epsilon}^{Y}(v)\right)
$$

Useful particular cases are when $S=\mathbf{R}, T=\mathbf{R}$ and $F:(s, t) \rightarrow$ st and when $S=\mathbf{R}^{N}, T=\mathbf{R}^{N}$ and $F$ is the dot product.

Proposition 3.2. If $u \in L^{1}\left(\mathbf{R}^{N}\right)$, then $\mathcal{T}_{\epsilon}^{Y}(u) \in L^{1}\left(\mathbf{R}^{n} \times Y\right)$ and

$$
\int_{\mathbf{R}^{N}} u(x) \mathrm{d} x=\frac{1}{|Y|} \int_{\mathbf{R}^{N} \times Y} \mathcal{T}_{\epsilon}^{Y}(u)(x, y) \mathrm{d} x \mathrm{~d} y .
$$

In particular, if $1 \leq p<+\infty$ and $u \in L^{p}\left(\mathbf{R}^{N}\right)$, then $\mathcal{T}_{\epsilon}^{Y}(u) \in L^{p}\left(\mathbf{R}^{N} \times Y\right)$, and

$$
\left\|\mathcal{T}_{\epsilon}^{Y}(u)\right\|_{L^{p}\left(\mathbf{R}^{N} \times Y\right)}=|Y|^{1 / p}\|u\|_{L^{p}\left(\mathbf{R}^{N}\right)} .
$$

Remark 18. In the sequel, a function which is defined on a set $A$ of $\mathbf{R}^{N}$, can be viewed as a function defined on $\mathbf{R}^{N}$, if we consider its extension by 0 outside of $A$.

The combination of Proposition 3.2 together with (3.1) yields: 
Proposition 3.3. Let $A \subset \mathbf{R}^{N}$ be measurable. If $u \in L^{1}(A)$, then $\mathcal{T}_{\epsilon}^{Y}\left(\chi_{A}\right) \mathcal{T}_{\epsilon}^{Y}(u)$ is well-defined on $\mathbf{R}^{N} \times \mathbf{R}^{N}, \mathcal{T}_{\epsilon}^{Y}\left(\chi_{A}\right) \mathcal{T}_{\epsilon}^{Y}(u) \in L^{1}\left(\mathbf{R}^{N} \times Y\right)$, and

$$
\int_{A} u(x) \mathrm{d} x=\frac{1}{|Y|} \int_{\mathbf{R}^{N} \times Y} \mathcal{T}_{\epsilon}^{Y}\left(\chi_{A}\right) \mathcal{T}_{\epsilon}^{Y}(u) \mathrm{d} x \mathrm{~d} y .
$$

Moreover, if $1 \leq p<+\infty$ and $u \in L^{p}(A)$, then $\mathcal{T}_{\epsilon}^{Y}\left(\chi_{A}\right) \mathcal{T}_{\epsilon}^{Y}(u)$ is welldefined on $\mathbf{R}^{N} \times \mathbf{R}^{N}, \mathcal{T}_{\epsilon}^{Y}\left(\chi_{A}\right) \mathcal{T}_{\epsilon}^{Y}(u) \in L^{p}\left(\mathbf{R}^{N} \times Y\right)$ and

$$
\left\|\mathcal{T}_{\epsilon}^{Y}\left(\chi_{A}\right) \mathcal{T}_{\epsilon}^{Y}(u)\right\|_{L^{p}\left(\mathbf{R}^{N} \times Y\right)}=|Y|^{1 / p}\|u\|_{L^{p}(A)} .
$$

Since the unfolding operator has a local action, it is natural to examine its effect on locally summable functions.

Proposition 3.4. For every $1 \leq p<\infty, \mathcal{T}_{\epsilon}^{Y}$ is a linear and continuous operator from $L_{\mathrm{loc}}^{p}\left(\mathbf{R}^{N}\right)$ to $L_{\mathrm{loc}}^{p}\left(\mathbf{R}^{N} \times \mathbf{R}^{N}\right)$.

We turn now to the $L_{\text {loc }}^{p}$ convergence properties for $1 \leq p<+\infty$.

Theorem 3.5. Let $\left(u_{\epsilon}\right)_{\epsilon}, u$ in $L_{\mathrm{loc}}^{p}\left(\mathbf{R}^{N}\right), 1 \leq p<+\infty$. If $u_{\epsilon} \rightarrow u$ strongly in $L_{\mathrm{loc}}^{p}\left(\mathbf{R}^{N}\right)$ then

$$
\mathcal{T}_{\epsilon}^{Y}\left(u_{\epsilon}\right) \rightarrow u \otimes 1 \quad \text { strongly in } L_{\mathrm{loc}}^{p}\left(\mathbf{R}^{N} \times \mathbf{R}^{N}\right) \text { as } \epsilon \rightarrow 0 .
$$

Global convergences follow easily.

Theorem 3.6. Let $A \subset \mathbf{R}^{N}$ be measurable $\left(u_{\epsilon}\right)_{\epsilon}, u$ in $L^{p}\left(\mathbf{R}^{N}\right), 1 \leq p<+\infty$. If $u_{\epsilon} \rightarrow u$ strongly in $L^{p}\left(\mathbf{R}^{N}\right)$, then

$$
\mathcal{T}_{\epsilon}^{Y}\left(\chi_{A}\right) \mathcal{T}_{\epsilon}^{Y}\left(u_{\epsilon}\right) \rightarrow\left(\chi_{A} u\right) \otimes 1 \text { strongly in } L^{p}\left(\mathbf{R}^{N} \times Y\right) \text { as } \epsilon \rightarrow 0,
$$

and

$$
\mathcal{T}_{\epsilon}^{Y}\left(u_{\epsilon}\right)_{\mid A \times Y} \rightarrow u \otimes 1 \text { strongly in } L^{p}(A \times Y) \text { as } \epsilon \rightarrow 0 .
$$

The following result states that the limit (if it exists) of an unfolded sequence is periodic.

Lemma 3.7. Let $u_{\epsilon} \in L_{\mathrm{loc}}^{1}\left(\mathbf{R}^{N}\right)\left(\operatorname{resp} L_{\mathrm{loc}}^{p}\left(\mathbf{R}^{N}\right)\right)$ and $\hat{u} \in L_{\mathrm{loc}}^{1}\left(\mathbf{R}^{N} \times \mathbf{R}^{N}\right)$ $\left(\operatorname{resp} L_{\mathrm{loc}}^{p}\left(\mathbf{R}^{N}\right)\right)$. If

$$
\left.\mathcal{T}_{\epsilon}^{Y}\left(u_{\epsilon}\right) \rightarrow \hat{u} *-\text { weakly in } \mathcal{M}\left(\mathbf{R}^{N} \times \mathbf{R}^{N}\right) \text {, (resp weakly in } L_{\mathrm{loc}}^{p}\left(\mathbf{R}^{N}\right)\right) \text {. }
$$

where $\mathcal{M}\left(\mathbf{R}^{N} \times \mathbf{R}^{N}\right)$ denotes the Radon measure space, then $\hat{u}$ is $Y$-periodic.

Next, we recall the properties of the unfolding operator applied on the gradient of some functions. If $u \in W_{\text {loc }}^{1, p}\left(\mathbf{R}^{N}\right)$ then by Proposition 3.4, 
$\mathcal{T}_{\epsilon}^{Y}(u) \in L_{\text {loc }}^{p}\left(\mathbf{R}^{N} \times \mathbf{R}^{N}\right)$ and $\mathcal{T}_{\epsilon}^{Y}(\nabla u) \in L_{\text {loc }}^{p}\left(\mathbf{R}^{N} \times \mathbf{R}^{N}\right)$. Moreover, for every test function $\varphi \in \mathcal{D}\left(\mathbf{R}^{N} \times \mathbf{R}^{N}\right)$

$$
\begin{gathered}
\int_{\mathbf{R}^{N} \times \mathbf{R}^{N}} \nabla_{y} \varphi \mathcal{T}_{\epsilon}^{Y}(u) \mathrm{d} x \mathrm{~d} y=\int_{\mathbf{R}^{N} \times \mathbf{R}^{N}} \nabla_{y} \varphi(x, y) u\left(\epsilon[x / \epsilon]_{Y}+\epsilon y\right) \mathrm{d} x \mathrm{~d} y \\
=-\int_{\mathbf{R}^{N} \times \mathbf{R}^{N}} \varphi(x, y) \epsilon \nabla u\left(\epsilon[x / \epsilon]_{Y}+\epsilon y\right) \mathrm{d} x \mathrm{~d} y \\
=-\int_{\mathbf{R}^{N} \times \mathbf{R}^{N}} \varphi \epsilon \mathcal{T}_{\epsilon}^{Y}(\nabla u) \mathrm{d} x \mathrm{~d} y
\end{gathered}
$$

Therefore $\mathcal{T}_{\epsilon}^{Y}(u)$ is weakly differentiable with respect to $y$, and

$$
\epsilon \mathcal{T}_{\epsilon}^{Y}(\nabla u)=\nabla_{y}\left(\mathcal{T}_{\epsilon}^{Y}(u)\right)
$$

The following result gives a relation between the limit of an unfolded sequence and the limit of the sequence:

Proposition 3.8. Let $\left(u_{\epsilon}\right)_{\epsilon}$ be a sequence of $L_{\mathrm{loc}}^{p}\left(\mathbf{R}^{N}\right)$ and let $u \in L_{\mathrm{loc}}^{p}\left(\mathbf{R}^{N}\right)$, $\hat{u} \in L_{\mathrm{loc}}^{p}\left(\mathbf{R}^{N} \times \mathbf{R}^{N}\right)$. Assume that $u_{\epsilon} \rightarrow u$ weakly in $L_{\mathrm{loc}}^{p}\left(\mathbf{R}^{N}\right)$ and $\mathcal{T}_{\epsilon}^{Y} u_{\epsilon} \rightarrow \hat{u}$ weakly in $L_{\mathrm{loc}}^{p}\left(\mathbf{R}^{N} \times \mathbf{R}^{N}\right)$, then

$$
u(x)=\frac{1}{|Y|} \int_{Y} \hat{u}(x, y) \mathrm{d} y .
$$

The following proposition is an important tool for the sequel.

Proposition 3.9. Let $\left(u_{\epsilon}\right)_{\epsilon}$ be a sequence of $W_{\mathrm{loc}}^{1, p}\left(\mathbf{R}^{N}\right)$ and let $\hat{u} \in L_{\mathrm{loc}}^{p}\left(\mathbf{R}^{N} ; \mathbf{R}^{N}\right)$. If $\left(u_{\epsilon}\right)_{\epsilon}$ is bounded in $L_{\mathrm{loc}}^{p}\left(\mathbf{R}^{N}\right),\left(\epsilon \nabla u_{\epsilon}\right)_{\epsilon}$ is bounded in $\left(L_{\mathrm{loc}}^{p}\left(\mathbf{R}^{N}\right)\right)^{N}$ and

$$
\mathcal{T}_{\epsilon}^{Y}\left(u_{\epsilon}\right) \rightarrow \hat{u} \text { weakly in } L_{\text {loc }}^{p}\left(\mathbf{R}^{N} \times \mathbf{R}^{N}\right) \text { as } \epsilon \rightarrow 0,
$$

then

$$
\epsilon \mathcal{T}_{\epsilon}^{Y}\left(\nabla u_{\epsilon}\right) \rightarrow \nabla_{y} \hat{u} \text { weakly in } L_{\mathrm{loc}}^{p}\left(\mathbf{R}^{N} \times \mathbf{R}^{N}\right) \text { as } \epsilon \rightarrow 0 .
$$

Moreover $\hat{u}$ is $Y$-periodic in $y$.

The following theorem is the main result.

Theorem 3.10. Let $\left(u_{\epsilon}\right)_{\epsilon}$ be a sequence of $W_{\mathrm{loc}}^{1, p}\left(\mathbf{R}^{N}\right)$ such that, $u_{\epsilon} \rightarrow u$ weakly in $W_{\text {loc }}^{1, p}\left(\mathbf{R}^{N}\right)$. Then, there exists a subsequence (not relabeled) and a function $\hat{u}$ in $L_{\mathrm{loc}}^{p}\left(\mathbf{R}^{N} ; W_{\mathrm{loc}}^{1, p}\left(\mathbf{R}^{N}\right)\right)$ such that the following convergence holds:

$$
\mathcal{T}_{\epsilon}^{Y}\left(\nabla u_{\epsilon}\right) \rightarrow \nabla u \otimes 1+\nabla_{y} \hat{u},
$$

weakly in $L_{\mathrm{loc}}^{p}\left(\mathbf{R}^{N} \times \mathbf{R}^{N}\right)$ as $\epsilon \rightarrow 0$. Additionally, $\hat{u}$ is $Y$-periodic.

When $\Omega \subsetneq \mathbf{R}^{N}, A: \Omega \rightarrow \mathfrak{M}\left(X \times X^{\prime}\right)$ is unfolded as follows:

Definition 3.11. Let $\Omega \subsetneq \mathbf{R}^{N}$ and $A: \Omega \rightarrow \mathfrak{M}\left(X \times X^{\prime}\right)$, first $A$ is extended by $\|\xi\|^{p-2} \xi$. This extension is still denoted by $A$. The unfolded graph $\mathcal{T}_{\epsilon}^{Y}(A)$ is now defined on $\mathbf{R}^{N} \times \mathbf{R}^{N}$. 


\subsection{Averaging operator}

Definition 3.12. Let $\epsilon>0, Y$ be a reference cell and $u$ in $L_{l o c}^{1}\left(\mathbf{R}^{N} \times \mathbf{R}^{N}\right)$, the averaging operator $\mathcal{U}_{\epsilon}^{Y}$ is defined as follows

$$
\mathcal{U}_{\epsilon}^{Y}(u)(x)=\frac{1}{|Y|} \int_{Y} u\left(\epsilon\left[\frac{x}{\epsilon}\right]_{Y}+\epsilon z, \epsilon\left\{\frac{x}{\epsilon}\right\}_{Y}\right) \mathrm{d} y .
$$

Proposition 3.13. For every $u \in L_{l o c}^{1}\left(\mathbf{R}^{N}\right)$, one has

$$
\mathcal{U}_{\epsilon}^{Y}\left(\mathcal{T}_{\epsilon}^{Y}(u)\right)(x)=u(x)
$$

Proposition 3.14. If $1 \leq p<\infty$ and $w \in L_{\mathrm{loc}}^{p}\left(\mathbf{R}^{N} \times \mathbf{R}^{N}\right)$ then

$$
\mathcal{T}_{\epsilon}^{Y}\left(\mathcal{U}_{\epsilon}^{Y}(w)\right) \rightarrow w
$$

strongly in $L_{\mathrm{loc}}^{p}\left(\mathbf{R}^{N} \times \mathbf{R}^{N}\right)$ as $\epsilon \rightarrow 0$.

We turn now to the $L^{p}$ locally convergence equivalence properties for $1 \leq p<+\infty$.

Theorem 3.15. Let $\left(u_{\epsilon}\right)_{\epsilon}$ in $L_{\mathrm{loc}}^{p}\left(\mathbf{R}^{N}\right)$ and $\tilde{u} \in L_{\mathrm{loc}}^{p}\left(\mathbf{R}^{N} \times \mathbf{R}^{N}\right), 1 \leq p<$ $+\infty$. The following strong convergences are equivalent:

i) $\quad \mathcal{T}_{\epsilon}^{Y}\left(u_{\epsilon}\right) \rightarrow \tilde{u} \quad$ strongly in $L_{\mathrm{loc}}^{p}\left(\mathbf{R}^{N} \times \mathbf{R}^{N}\right)$ as $\epsilon \rightarrow 0$,

ii) $\quad u_{\epsilon}-\mathcal{U}_{\epsilon}^{Y}(\tilde{u}) \rightarrow 0 \quad$ strongly in $L_{\mathrm{loc}}^{p}\left(\mathbf{R}^{N}\right)$ as $\epsilon \rightarrow 0$.

\section{Homogenization results}

In this section we state our main homogenization result. First we give the result and the proof of the convergence. Then we study the properties of the homogenized operator. Finally we give some energy convergence and corrector result.

\subsection{Convergence results}

Theorem 4.1. Assume $1<p<\infty, p^{-1}+q^{-1}=1, m_{\epsilon}$ in $L^{1}(\Omega), \alpha>0$ and let $\Omega$ be a bounded open set of $\mathbf{R}^{N}$. Let $A_{\epsilon} \in \mathfrak{M}\left(\Omega, \mathbf{R}^{N}, p, \alpha, m_{\epsilon}\right)$ for every $\epsilon>0$.

Assume that there exists a cell $Y \subset \mathbf{R}^{N}$, a measurable $B: \Omega \times Y \rightarrow$ $\mathfrak{M}\left(\mathbf{R}^{N} \times \mathbf{R}^{N}\right)$ and $m \in L^{1}(\Omega \times Y)$ such that for almost every $(x, y) \in \Omega \times Y$,

$$
\mathcal{T}_{\epsilon}^{Y}\left(A_{\epsilon}\right)(x, y) \longmapsto B(x, y)
$$

and $\mathcal{T}_{\epsilon}\left(m_{\epsilon}\right)$ converges to $m$ strongly in $L^{1}(\Omega \times Y)$ as $\epsilon$ goes to 0 .

Assume $f_{\epsilon} \rightarrow f_{0}$ strongly in $W^{-1, q}(\Omega)$ as $\epsilon \rightarrow 0$. 
Consider a (not necessarily unique) solution $u_{\epsilon} \in W_{0}^{1, p}(\Omega)$ of the problem

$$
\left\{\begin{array}{l}
-\operatorname{div} d_{\epsilon}=f_{\epsilon} \text { in } \mathcal{D}^{\prime}(\Omega), \\
\left(\nabla u_{\epsilon}(x), d_{\epsilon}(x)\right) \in A_{\epsilon}(x) \\
u_{\epsilon} \in W_{0}^{1, p}(\Omega)
\end{array}\right.
$$

Then, the family $\left(u_{\epsilon}, d_{\epsilon}\right)_{\epsilon>0}$ is weakly compact in $W_{0}^{1, p}(\Omega) \times L^{q}\left(\Omega ; \mathbf{R}^{N}\right)$. Moreover, if $\left(u_{0}, d_{0}\right)$ is one of its limit points, i.e. there is $\left(\epsilon_{n}\right)_{n \geq 1}$ such that $\epsilon_{n} \rightarrow 0$

$$
\begin{aligned}
& u_{\epsilon_{n}} \rightarrow u_{0} \text { weakly in } W_{0}^{1, p}(\Omega), \\
& d_{\epsilon_{n}} \rightarrow d_{0} \text { weakly in } L^{q}\left(\Omega ; \mathbf{R}^{N}\right),
\end{aligned}
$$

then

$$
\left\{\begin{array}{l}
-\operatorname{div} d_{0}=f_{0} \text { in } \mathcal{D}^{\prime}(\Omega) \\
\left(\nabla u_{0}(x), d_{0}(x)\right) \in A(x) \\
u_{0} \in W_{0}^{1, p}(\Omega)
\end{array}\right.
$$

where, for almost every $x \in \Omega$,

$$
\begin{gathered}
A(x)=\left\{(\xi, \eta) \in \mathbf{R}^{N} \times \mathbf{R}^{N}: \exists(\hat{u}, \hat{d}) \in W_{\mathrm{per}}^{1, p}(Y) \times L^{q}\left(Y ; \mathbf{R}^{N}\right),\right. \\
\frac{1}{|Y|} \int_{Y} \hat{u}(y) \mathrm{d} y=0, \frac{1}{|Y|} \int_{Y} \hat{d}(y) \mathrm{d} y=0, \\
(\xi+\nabla \hat{u}(y), \eta+\hat{d}(y)) \in B(x, y) \text { for a.e. } y \in Y \\
\text { and } \left.-\operatorname{div} \hat{d}=0 \text { in }\left(\mathcal{C}_{\mathrm{per}}^{\infty}\right)^{\prime}(Y)\right\} .
\end{gathered}
$$

Moreover $A_{0} \in \mathfrak{M}\left(\Omega, \mathbf{R}^{N}, p, \alpha, \bar{m}\right)$, where

$$
\bar{m}(x)=\frac{1}{|Y|} \int_{Y} m(x, y) d y .
$$

Remark 19. Theorem 4.1 applies to the particular case where $A_{\epsilon}(x)=$ $A(x / \epsilon)$, provided $A \in \mathfrak{M}\left(\Omega, \mathbf{R}^{N}, p, \alpha, m\right)$ and $m \in L_{\text {loc }}^{1}\left(\mathbf{R}^{N}\right)$ are $Y$-periodic. In such a case, $\mathcal{T}_{\epsilon}^{Y}\left(A_{\epsilon}\right)(x, y)=A(y)$, so that (4.1) immediately follows. Note that this case was treated by [8].

Remark 20. The family of maximal monotone graphs $A_{0}(x)$ defined in the previous Theorem 4.1 is the $H$-limit (see [18] and [20]) of the sequence of graphs $A_{\epsilon}(x)$. Our results are in accordance with the fact that $\mathfrak{M}\left(\Omega, \mathbf{R}^{N}, p, \alpha, m\right)$ is closed under $H$-limit [7].

By Theorem 3.11 and Remark 3.12 in [7], for every $\left(u_{0}, d_{0}\right)$ in $W_{0}^{1, p}(\Omega) \times$ $L^{q}\left(\Omega ; \mathbf{R}^{N}\right),\left(\nabla u_{0}(x), d_{0}(x)\right)$ belongs to $A(x)$ almost everywhere. There exists $\left(u_{\epsilon}, d_{\epsilon}\right)_{\epsilon>0} \in W_{0}^{1, p}(\Omega) \times L^{q}\left(\Omega ; \mathbf{R}^{N}\right)$ such that $\left(u_{\epsilon}(x), d_{\epsilon}(x)\right) \in A_{\epsilon}(x), u_{\epsilon} \rightarrow u$ weakly in $W_{0}^{1, p}(\Omega)$ and $\operatorname{div} d_{\epsilon} \rightarrow \operatorname{div} d_{0}$ strongly in $W^{-1, q}\left(\Omega ; \mathbf{R}^{N}\right)$. 
Remark 21. For every $\epsilon>0$, the existence of (at least) one solution of problem (4.2) follows from [13] or [7]. Indeed, using [13], one should check that there exists $\bar{d}_{\epsilon} \in L^{q}\left(\Omega ; \mathbf{R}^{N}\right)$ such that $\left(0, \bar{d}_{\epsilon}(x)\right) \in A_{\epsilon}(x)$. This is always the case by Corollary 2.15 .

Proof of Theorem 4.1. First we establish the weak compactness of the unfolded sequences $\left(\mathcal{T}_{\epsilon}\left(\nabla u_{\epsilon}\right), \mathcal{T}_{\epsilon}\left(d_{\epsilon}\right)\right)$. Extend $u_{\epsilon}$ and $d_{\epsilon}$ by 0 outside of $\Omega$. From inequality (2.6) and taking $u_{\epsilon}$ as a test function in equation (4.2), we deduce that the sequences $\left(u_{\epsilon}\right)_{\epsilon}$ is bounded in $W^{1, p}\left(\mathbf{R}^{N}\right)$ and the sequence $\left(d_{\epsilon}\right)_{\epsilon}$ is bounded in $L^{q}\left(\mathbf{R}^{N} ; \mathbf{R}^{N}\right)$. Hence they are weakly compact.

Assume now $u_{\epsilon_{n}} \rightarrow u_{0}$ weakly in $W_{0}^{1, p}(\Omega)$ and $d_{\epsilon_{n}} \rightarrow d_{0}$ in $L^{q}\left(\mathbf{R}^{N} ; \mathbf{R}^{N}\right)$. By Theorem 3.10, there is $\hat{u} \in L^{p}\left(\Omega ; W_{\text {per }}^{1, p}(\Omega)\right)$ such that

$$
\mathcal{T}_{\epsilon_{n}}^{Y}\left(\nabla u_{\epsilon_{n}}\right) \rightarrow \nabla_{x} u_{0}+\nabla_{y} \hat{u}
$$

weakly in $L^{p}\left(\mathbf{R}^{N} \times Y ; \mathbf{R}^{N}\right)$. The sequence $\mathcal{T}_{\epsilon_{n}}^{Y}\left(d_{\epsilon_{n}}\right)$ is bounded in $L^{q}\left(\mathbf{R}^{N} \times\right.$ $Y)$. There exists $\eta \in L^{q}\left(\mathbf{R}^{N} \times Y\right)$, so that, up to a subsequence, $\mathcal{T}_{\epsilon_{n}}^{Y}\left(d_{\epsilon_{n}}\right) \rightarrow$ $\eta$. Then $d_{0}=\frac{1}{|Y|} \int_{Y} \eta(y) \mathrm{d} y$ so setting $\hat{d}=\eta-\frac{1}{|Y|} \int_{Y} \eta(y) \mathrm{d} y$, one has

$$
\mathcal{T}_{\epsilon_{n}}^{Y}\left(d_{\epsilon_{n}}\right) \rightarrow d_{0}+\hat{d} \text { weakly in } L^{q}\left(\mathbf{R}^{N} \times Y\right) .
$$

Let $\varphi \in \mathcal{C}_{c}^{\infty}(\Omega)$. By the weak convergence of $d_{\epsilon_{n}}$ and the strong convergence of $f_{\epsilon_{n}}$, letting $n \rightarrow \infty$ in (4.2) yields

$$
\int_{\Omega}\left\langle d_{0}(x), \nabla \varphi(x)\right\rangle \mathrm{d} x=\int_{\Omega} f_{0} \varphi \mathrm{d} x
$$

i.e. $-\operatorname{div} d_{0}=f_{0}$ in $\mathcal{D}^{\prime}(Y)$. Next, let $\varphi \in \mathcal{D}(\Omega)$ and $\psi \in C^{\infty}\left(\mathbf{R}^{N}\right)$, such that $\psi$ is $Y$-periodic. Defining $\varphi_{\epsilon_{n}}(x)=\epsilon_{n} \varphi(x) \psi\left(x / \epsilon_{n}\right)$, one has, by the equation 4.2 and Proposition 3.3,

$$
\frac{1}{|Y|} \int_{\mathbf{R}^{N} \times Y}\left\langle\mathcal{T}_{\epsilon_{n}}^{Y}\left(d_{\epsilon_{n}}\right), \mathcal{T}_{\epsilon_{n}}^{Y}\left(\nabla \varphi_{\epsilon_{n}}\right)\right\rangle \mathrm{d} x \mathrm{~d} y=\int_{\Omega} f_{\epsilon_{n}} \varphi_{\epsilon_{n}} \mathrm{~d} x
$$

Letting $\epsilon_{n} \rightarrow 0$, one has $\varphi_{\epsilon_{n}} \rightarrow 0$ weakly in $W^{1, p}(\Omega)$ and $\mathcal{T}_{\epsilon_{n}}^{Y}\left(\nabla \varphi_{\epsilon_{n}}\right) \rightarrow$ $\varphi(x) \nabla \psi(y)$ strongly in $L^{p}\left(\Omega ; W_{\text {per }}^{1, p}(Y)\right)$, so that

$$
\frac{1}{|Y|} \int_{\Omega \times Y}\left\langle d_{0}(x)+\hat{d}(x, y), \varphi(x) \nabla \psi(y)\right\rangle \mathrm{d} x \mathrm{~d} y=0 .
$$

Since $\varphi$ is arbitrary, one concludes that for almost every $x \in \Omega$, for every $\psi \in W_{\text {per }}^{1, p}(Y)$,

$$
\frac{1}{|Y|} \int_{Y}\left\langle\hat{d}(x, y), \nabla_{y} \psi(y)\right\rangle \mathrm{d} y=0,
$$

i.e. $-\operatorname{div} \hat{d}(x, \cdot)=0$ in $\left(\mathcal{C}_{\text {per }}^{\infty}\right)^{\prime}(Y)$. 
Letting $B_{\epsilon_{n}}=\mathcal{T}_{\epsilon_{n}}^{Y}\left(A_{\epsilon_{n}}\right)$, one has by Corollary $2.17, \mathcal{B}_{\epsilon_{n}} \longmapsto \mathcal{B}$ as $n \rightarrow \infty$. One also has

$$
\begin{aligned}
\int_{\mathbf{R}^{N} \times Y}\left\langle\mathcal{T}_{\epsilon_{n}}^{Y}\left(d_{\epsilon_{n}}\right), \mathcal{T}_{\epsilon_{n}}^{Y}\left(\nabla u_{\epsilon_{n}}\right)\right\rangle & =\int_{\Omega} f_{\epsilon_{n}} u_{\epsilon_{n}} \mathrm{~d} x \\
\rightarrow \int_{\Omega} f_{0} u_{0} \mathrm{~d} x & =\int_{\mathbf{R}^{N} \times Y}\left\langle d_{0}+\hat{d}, \nabla_{x} u_{0}+\nabla_{y} \hat{u}\right\rangle \mathrm{d} x \mathrm{~d} y,
\end{aligned}
$$

where the last equality comes from (4.5) and (4.6), together with the fact that $\frac{1}{|Y|} \int_{Y} \nabla_{y} \hat{u} \mathrm{~d} y=0$ and $\frac{1}{|Y|} \int_{Y} \hat{d} \mathrm{~d} y=0$. By Theorem $2.8,\left(\nabla_{x} u_{0}(x)+\right.$ $\left.\nabla_{y} \hat{u}(x, y), d_{0}(x)+\hat{d}(x, y)\right) \in B(x, y)$. This, together with (4.6), implies that $\left(\nabla u_{0}(x), d_{0}(x)\right) \in A(x)$ for almost every $x \in \Omega$.

By Proposition 4.2 below, for almost every $x \in \Omega, A_{0}(x)$ is maximal monotone and satisfies (2.6). Its measurability comes from Lusin's Theorem, together with Proposition 4.4. Therefore, $A \in \mathfrak{M}\left(\Omega, \mathbf{R}^{N}, p, \alpha, \bar{m}\right)$ as claimed.

Remark 22. The previous proof easily extends to different boundary conditions for which a variational formulation holds.

Remark 23. The proof can be extended to the reiterated case as in [16].

\subsection{Properties of the homogenized graph}

In this section, we prove some properties of the homogenized graph $A$.

Proposition 4.2. Let $\alpha>0, m \in L^{1}(\Omega)$, and $B \in \mathfrak{M}\left(Y, \mathbf{R}^{N}, p, \alpha, m\right)$. Define

$$
\begin{gathered}
A=\left\{(\xi, \eta) \in \mathbf{R}^{N} \times \mathbf{R}^{N}: \exists(\hat{u}, \hat{d}) \in W_{\mathrm{per}}^{1, p}(Y) \times L^{q}\left(Y ; \mathbf{R}^{N}\right),\right. \\
\quad \frac{1}{|Y|} \int_{Y} \hat{u}(y) \mathrm{d} y=0, \frac{1}{|Y|} \int_{Y} \hat{d}(y) \mathrm{d} y=0 \\
(\xi+\nabla \hat{u}(y), \eta+\hat{d}(y)) \in B(y) \text { a.e. } y \in Y \\
\text { and } \left.-\operatorname{div} \hat{d}=0 \text { in }\left(\mathcal{C}_{\mathrm{per}}^{\infty}\right)^{\prime}(Y),\right\},
\end{gathered}
$$

then $A$ belongs to $\mathfrak{M}\left(\mathbf{R}^{N} \times \mathbf{R}^{N}\right)$, and for every $(\xi, \eta) \in A$,

$$
\alpha\left(\frac{\|\xi\|^{p}}{p}+\frac{\|\eta\|^{q}}{q}\right) \leq\langle\eta, \xi\rangle+\frac{1}{|Y|} \int_{Y} m(y) y .
$$

Proof. First, one checks the monotonicity of $A$. Let $\left(\xi_{i}, \eta_{i}\right) \in A$, for $i=1,2$ and let $\hat{u}_{i}$ and $\hat{d}_{i}$ be associated to $\left(\xi_{i}, \eta_{i}\right)$ by the definition. One has then

$$
\begin{aligned}
& \left\langle\eta_{1}-\eta_{2}, \xi_{1}-\xi_{2}\right\rangle \\
& \quad=\frac{1}{|Y|} \int_{Y}\left\langle\eta_{1}+\hat{d}_{1}(y)-\eta_{2}-\hat{d}_{2}(y), \xi_{1}+\nabla \hat{u}_{1}(y)-\xi_{2}-\nabla \hat{u}_{2}(y)\right\rangle \mathrm{d} y
\end{aligned}
$$


By the monotonicity of $B$, the right-hand side is nonnegative. Therefore $A$ is monotone.

By Theorem 2.18 the maximality of $A$ will follow from the surjectivity of $A+F_{p}$. For a.e. $y \in Y$ and for every $\epsilon>0$ and $\lambda>0$, set

$$
B_{\epsilon, \lambda}(y)=\epsilon F_{p}(y)+B(y)_{\lambda, p},
$$

according to Definition 2.19. For almost every $y \in Y, B_{\epsilon, \lambda}(y)$ is maximal monotone, continuous and coercive. We are now going to build an approximation $B_{\epsilon, \lambda}$ of $B$ and then let $(\epsilon, \lambda)$ go to zero. For $\epsilon$ and $\lambda$ small enough, the fact that $B_{\epsilon, \lambda} \in \mathfrak{M}\left(Y, \mathbf{R}^{N}, p, \alpha / 2, m\right)$ follows from Lemma 4.3 below.

Since $B_{\epsilon, \lambda}$ satisfies the hypotheses of Leray-Lions [14], we know that for every $\xi \in \mathbf{R}^{N}$ there exists a unique $\hat{u} \in W_{\text {per }}^{1, p}(Y)$ such that

$$
\left\{\begin{array}{l}
\frac{1}{|Y|} \int_{Y} \hat{u}(y) \mathrm{d} y=0 \\
-\operatorname{div} B_{\epsilon, \lambda}(y)(\nabla \hat{u}(y)+\xi)=0 \text { in }\left(\mathcal{C}_{\mathrm{per}}^{\infty}\right)^{\prime}(Y) .
\end{array}\right.
$$

Define $\hat{d}=B_{\epsilon, \lambda}(y)(\nabla \hat{u}(y)+\xi)$ and

$$
g(\xi)=-\frac{1}{|Y|} \int_{Y} B_{\epsilon, \lambda}(y)(\nabla \hat{u}(y)+\xi) \mathrm{d} y .
$$

By (2.6), one has

$$
\begin{aligned}
\frac{\alpha}{2} \frac{1}{|Y|} \int_{Y} \frac{\|\nabla \hat{u}+\xi\|^{p}}{p}+\frac{\|\hat{d}\|^{q}}{q} \mathrm{~d} y & \leq \frac{1}{|Y|} \int_{Y}\langle\hat{d}, \nabla \hat{u}+\xi\rangle \mathrm{d} y+\frac{1}{|Y|} \int_{Y} m \mathrm{~d} y \\
& =-\langle\xi, g(\xi)\rangle+\frac{1}{|Y|} \int_{Y} m \mathrm{~d} y .
\end{aligned}
$$

By Jensen's inequality, since $\int_{Y} \nabla \hat{u} \mathrm{~d} y=0$,

$$
\frac{\alpha}{2}\left(\frac{\|\xi\|^{p}}{p}+\frac{\|g(\xi)\|^{q}}{q}\right) \leq-\langle\xi, g(\xi)\rangle+\int_{Y} m \mathrm{~d} y .
$$

From (4.12) follows an explicit bound of $\|g(\xi)\|$ in terms $\|\xi\|$.

Let us show that $g: \mathbf{R}^{N} \rightarrow \mathbf{R}^{N}$ is continuous. Assume $\xi_{n} \rightarrow \xi_{0}$ as $n \rightarrow \infty$. Let $\hat{u}_{n}$ be the unique solution of problem (4.10) associated to $\xi_{n}$, and let $\hat{d}_{n}=B_{\epsilon, \lambda}(y)\left(\nabla \hat{u}_{n}(y)+\xi\right)$. By 4.12 the sequences $\left(\hat{d}_{n}\right)$ and $\left(\hat{u}_{n}\right)$ are bounded. Therefore there exists $\left(\hat{d}_{0}^{\prime}, \hat{u}_{0}^{\prime}\right)$ such that, up to a subsequence,

$$
\begin{gathered}
\hat{d}_{n} \rightarrow \hat{d}_{0}^{\prime} \text { weakly in } L^{q}(Y), \\
\hat{u}_{n} \rightarrow \hat{u}_{0}^{\prime} \text { weakly in } W_{\text {per }}^{1, p}(Y) .
\end{gathered}
$$

Recalling that $-\operatorname{div} \hat{d}_{n}=0,-\operatorname{div} \hat{d}_{0}^{\prime}=0$, in $\left(\mathcal{C}_{\text {per }}^{\infty}\right)^{\prime}(Y)$ together with $(4.14)$ and $\xi_{n} \rightarrow \xi_{0}$, this gives

$$
\begin{aligned}
\int_{Y}\left\langle\hat{d}_{n}, \nabla \hat{u}_{n}+\xi_{n}\right\rangle \mathrm{d} y=\int_{Y}\left\langle\hat{d}_{n}, \xi_{n}\right\rangle \mathrm{d} y & \\
& \rightarrow \int_{Y}\left\langle\hat{d}_{0}^{\prime}, \xi_{0}\right\rangle \mathrm{d} y=\int_{Y}\left\langle\hat{d}_{0}^{\prime}, \nabla \hat{u}_{0}^{\prime}+\xi_{0}\right\rangle \mathrm{d} y .
\end{aligned}
$$


By Lemma 2.5, we deduce that $\left(\nabla \hat{u}_{0}^{\prime}(y)+\xi_{0}, \hat{d}_{0}^{\prime}(y)\right) \in B_{\epsilon, \lambda}(y)$. By the uniqueness of the solution of problem $(4.10),\left(\hat{u}_{0}^{\prime}, \hat{d}_{0}^{\prime}\right)=\left(\hat{u}_{0}, \hat{d}_{0}\right)$. In particular

$$
g\left(\xi_{0}\right)=\frac{1}{|Y|} \int_{Y} \hat{d}_{0} \mathrm{~d} y=\lim _{n \rightarrow+\infty} \frac{1}{|Y|} \int_{Y} \hat{d}_{n} \mathrm{~d} y=\lim _{n \rightarrow+\infty} g\left(\xi_{n}\right) .
$$

Let us now check the hypothesis of Schaefer's fixed point theorem (see [19]). First, $g$ is continuous. Next, assume there are $\mu>1$ and $\xi \in \mathbf{R}^{N}$ such that $g(\xi)+\eta=\mu \xi$ and $\mu>1$. By (4.13) one has then

$$
\alpha^{\prime}\left(\frac{\|\xi\|^{p}}{p}+\frac{\|g(\xi)\|^{q}}{q}\right) \leq-\langle\xi, g(\xi)\rangle+\int_{Y} m \mathrm{~d} y \leq\langle\xi, \eta\rangle+\int_{Y} m \mathrm{~d} y .
$$

and therefore $\|\xi\| \leq C$, where $C$ depends only on $p, \alpha$ and $m$. The previous statement means that for every $\eta \in \mathbf{R}^{N}$, there is $\xi \in \mathbf{R}^{N}$ such that $\xi=$ $g(\xi)+\eta$ by Schaefer's fixed point theorem.

By definition of $g$, there exists $(\hat{u}, \hat{d})$ in $W_{\text {per }}^{1, p}(Y) \times L^{q}(Y)$ such that

$$
\left\{\begin{array}{l}
-\operatorname{div} \hat{d}=0 \text { in }\left(\mathcal{C}_{\text {per }}^{\infty}\right)^{\prime}(Y) \\
\hat{d}=B_{\epsilon, \lambda}(y)(\nabla \hat{u}(y)+\xi) \mathrm{d} y \\
\eta=\xi+\frac{1}{|Y|} \int_{Y} B_{\epsilon, \lambda}(y)(\nabla \hat{u}(y)+\xi) \mathrm{d} y
\end{array}\right.
$$

Consider now sequences $\epsilon_{n} \rightarrow 0, \lambda_{n} \rightarrow 0$ as $n \rightarrow \infty$. Let $\hat{u}_{n}, \hat{d}_{n}, \xi_{n}$ be defined by (4.17), for $n$ associated to $\epsilon_{n}, \lambda_{n}$. By (4.12) and (4.13), the sequences $\left(\beta_{n}\right),\left(\hat{u}_{n}\right)$ and $\left(\hat{d}_{n}\right)$ are bounded in $\mathbf{R}^{N}, W_{\text {per }}^{1, p}(Y)$ and $L^{q}(Y)$ respectively. Up to subsequences, it follows that

$$
\begin{gathered}
\xi_{n} \rightarrow \xi_{0} \text { in } \mathbf{R}^{N}, \\
\hat{u}_{n} \rightarrow \hat{u}_{0} \text { weakly in } W_{\text {per }}^{1, p}(Y), \\
\hat{d}_{n} \rightarrow \hat{d}_{0} \text { weakly in } L^{q}(Y) .
\end{gathered}
$$

Furthermore, recalling Corollary $2.17, \mathcal{B}_{\epsilon_{n}, \lambda_{n}} \longmapsto \mathcal{B}$, as $n \rightarrow+\infty$. that

Recalling that $\int_{Y}\left\langle\hat{d}_{n}, \nabla \varphi\right\rangle \mathrm{d} y=0$, for every $\varphi \in W_{\text {per }}^{1, p}(Y)$, we deduce

$$
\int_{Y}\left\langle\hat{d}_{n}, \nabla \hat{u}_{n}+\xi_{n}\right\rangle \mathrm{d} y=\int_{Y}\left\langle\hat{d}_{n}, \xi_{n}\right\rangle \mathrm{d} y
$$

and since $\xi_{n} \rightarrow \xi_{0}, \hat{d}_{n} \rightarrow \hat{d}_{0}$, passing to the limit as $n \rightarrow \infty$, we deduce that

$$
\int_{Y}\left\langle\hat{d}_{0}, \nabla \hat{u}_{0}+\xi_{0}\right\rangle \mathrm{d} y=\int_{Y}\left\langle\hat{d}_{0}, \xi_{0}\right\rangle \mathrm{d} y
$$

Consequently, by Theorem 2.8 , we have $\left(\nabla \hat{u}_{0}(y)+\xi_{0}, \hat{d}_{0}(y)\right) \in B(y)$ for a.e. $y \in Y,-\operatorname{div} \hat{d}_{0}=0$ in $\left(\mathcal{C}_{\text {per }}^{\infty}\right)^{\prime}(Y)$ and $\int_{Y} \hat{d}_{0} \mathrm{~d} y=\xi_{0}$. This means that $A \xi_{0}+\xi_{0}=\eta$, and therefore $A+F$ is surjective. 
In order to prove $(4.9)$, let $(\xi, \eta) \in A$, and let $\hat{u}, \hat{d}$ be given by the definition of $A$. Since $\frac{1}{|Y|} \int_{Y} \nabla \hat{u} \mathrm{~d} y=0$ by periodicity and $\frac{1}{|Y|} \int_{Y} \hat{d}(y) \mathrm{d} y=0$, we have, by Jensen's inequality,

$$
\alpha\left(\frac{\|\xi\|^{p}}{p}+\frac{\|\eta\|^{q}}{q}\right) \leq \frac{\alpha}{|Y|} \int_{Y} \frac{\|\xi+\nabla \hat{u}(y)\|^{p}}{p}+\frac{\|\eta+\hat{d}(y)\|^{q}}{q} \mathrm{~d} y,
$$

Together with (2.6), this yields

$$
\alpha\left(\frac{\|\xi\|^{p}}{p}+\frac{\|\eta\|^{q}}{q}\right) \leq \frac{1}{|Y|} \int_{Y}\langle\eta+\hat{d}, \xi+\nabla \hat{u}\rangle \mathrm{d} y+\frac{1}{|Y|} \int_{Y} m \mathrm{~d} y .
$$

Using again that $\frac{1}{|Y|} \int_{Y} \nabla \hat{u} \mathrm{~d} y=0$ and $\frac{1}{|Y|} \int_{Y} \hat{d}(y) \mathrm{d} y=0$ together with $-\operatorname{div} \hat{d}=0$ in $\left(\mathcal{C}_{\text {per }}^{\infty}\right)^{\prime}(Y)$, one obtains (4.9).

Lemma 4.3. Let $\alpha>0$. For every $\delta>0$, there is $\epsilon_{0}>0$ and $\lambda_{0}>0$ such that if $m \in \mathbf{R}$ and $B \in \mathfrak{M}\left(X \times X^{\prime}\right)$ are such that for every $(\xi, \eta) \in B$,

$$
\alpha\left(\frac{\|\xi\|^{p}}{p}+\frac{\|\eta\|^{q}}{q}\right) \leq\langle\eta, \xi\rangle+m
$$

then for every $\xi \in X, 0<\epsilon<\epsilon_{0}$ and $0<\lambda<\lambda_{0}$,

$$
(\alpha-\delta)\left(\frac{\|\xi\|^{p}}{p}+\frac{\left\|B_{\lambda, p} \xi+\epsilon F_{p}(\xi)\right\|^{q}}{q}\right) \leq\left\langle B_{\lambda, p} \xi+\epsilon F_{p}(\xi), \xi\right\rangle+m
$$

Proof. Let $\xi \in X, \eta=\epsilon F_{p}(\xi)+B_{\lambda, p} \xi$ and $\zeta=J_{\lambda, p}^{B} \xi$. One has $\left(\zeta, \eta-\epsilon F_{p}(\xi)\right) \epsilon$ $B$. Therefore

$$
\alpha\left(\frac{\|\zeta\|^{p}}{p}+\frac{\left\|\eta-\epsilon F_{p}(\xi)\right\|^{q}}{q}\right) \leq\left\langle\eta-\epsilon F_{p}(\xi), \zeta\right\rangle+m .
$$

Since $F_{p}(\xi-\zeta)=\lambda^{p-1}\left(\eta-\epsilon F_{p}(\xi)\right)$, one can develop

$$
\left\langle\eta-\epsilon F_{p}(\xi), \zeta\right\rangle=\langle\eta, \xi\rangle-\lambda^{1-p}\|\xi-\zeta\|^{p}-\epsilon^{1-q}\left\|\epsilon F_{p}(\xi)\right\|^{q} .
$$

Bringing this together gives

$$
\alpha\left(\frac{\|\zeta\|^{p}}{p}+\frac{\|\xi-\zeta\|^{p}}{\alpha \lambda^{p-1}}+\frac{\left\|\eta-\epsilon F_{p}(\xi)\right\|^{q}}{q}+\frac{\left\|\epsilon F_{p}(\xi)\right\|^{q}}{\alpha \epsilon^{q-1}}\right) \leq\langle\eta, \xi\rangle+m .
$$

By convexity, for every $\mu \in(0,1)$,

$$
\begin{gathered}
\|\xi\|^{p} \leq \frac{\|\zeta\|^{p}}{\mu^{p-1}}+\frac{\|\xi-\zeta\|^{p}}{(1-\mu)^{p-1}}, \\
\|\eta\|^{q} \leq \frac{\left\|F_{p}(\xi)\right\|^{q}}{\mu^{q-1}}+\frac{\left\|F_{p}(\xi)-\eta\right\|^{p}}{(1-\mu)^{q-1}} .
\end{gathered}
$$

Choose $\mu \in(0,1)$ such that $(\alpha-\delta) \max \left(\mu^{p-1}, \mu^{q-1}\right) \leq 1$. The required inequality holds with $\lambda_{0}=(p / \alpha)^{1 /(p-1)}(1-\mu)$ and $\epsilon_{0}=(q / \alpha)^{1 /(q-1)}(1-$ q). 
Let us now establish the following result which gives the measurability of $A(x)$ (using Lusin's theorem) in Theorem 4.1.

Proposition 4.4. Let $\alpha>0, m_{n} \in L^{1}(\Omega)$. Let $B_{n} \in \mathfrak{M}\left(Y, \mathbf{R}^{N}, p, \alpha, m_{n}\right)$ and $B \in \mathfrak{M}\left(\mathbf{R}^{N} \times \mathbf{R}^{N}\right)$ be measurable. Let $A^{n}$ and $A$ be the graphs associated by (4.8). If $m_{n} \rightarrow m$ strongly in $L^{1}(\Omega)$ and $B^{n}(y) \longmapsto B(y)$, then $A^{n} \longmapsto A$, as $n \rightarrow \infty$.

Proof. By Theorem 2.9, it is sufficient to show that for every $\zeta \in \mathbf{R}, A_{1}^{n} \zeta \rightarrow$ $A_{1} \zeta$. Let $\left(\xi_{n}, \eta_{n}\right)=\left(J_{1}^{A^{n}} \zeta, A_{1}^{n} \zeta\right)$. Since $F$ is the identity mapping, one has $\xi_{n}+\eta_{n}=\zeta$ and $\left(\xi_{n}, \eta_{n}\right) \in A^{n}$. By definition of $A^{n}$, there exists $\left(\hat{u}_{n}, \hat{d}_{n}\right) \in$ $W_{\text {per }}^{1, p}(Y) \times L^{q}\left(Y ; \mathbf{R}^{N}\right)$ associated to $A^{n}$ by the definition (4.8).

By (2.6), one has

$$
\alpha \int_{Y} \frac{\left\|\nabla \hat{u}_{n}+\xi_{n}\right\|^{p}}{p}+\frac{\left\|\hat{d}_{n}+\eta_{n}\right\|^{q}}{q} \mathrm{~d} y \leq\left\langle\eta_{n}, \xi_{n}\right\rangle+\int_{Y} m_{n} \mathrm{~d} y .
$$

By Jensen's inequality and since $\eta_{n}=\zeta-\xi_{n}$,

$$
\alpha\left(\frac{\left\|\xi_{n}\right\|^{p}}{p}+\frac{\left\|\eta_{n}\right\|^{q}}{q}\right) \leq\left\langle\zeta, \xi_{n}\right\rangle+\int_{Y} m_{n} \mathrm{~d} y,
$$

so that the sequences $\xi_{n}$ and $\eta_{n}$ are bounded in $\mathbf{R}^{N}$. By (4.18), the sequences $\hat{d}_{n}$, and $\hat{u}_{n}$ are bounded in $L^{q}(Y)$ and $W_{\text {per }}^{1, p}(Y)$ respectively. Up to a subsequence, $\left(\xi_{n}, \eta_{n}\right) \rightarrow(\xi, \eta)$ in $\mathbf{R}^{N} \times \mathbf{R}^{N}$ and $\left(\hat{u}_{n}, \hat{d}_{n}\right) \rightarrow(\hat{u}, \hat{d})$ weakly in $W_{\text {per }}^{1, p}(Y) \times L^{q}(Y)$. In particular, $\frac{1}{|Y|} \int_{Y} \nabla \hat{u} \mathrm{~d} y=0, \frac{1}{|Y|} \int_{Y} \hat{d} \mathrm{~d} y=0$ and $-\operatorname{div} \hat{d}=0$ in $\left(\mathcal{C}_{\text {per }}^{\infty}\right)^{\prime}(Y)$. Therefore,

$$
\int_{Y}\left\langle\hat{d}_{n}+\eta_{n}, \nabla \hat{u}_{n}+\xi_{n}\right\rangle \mathrm{d} y=\left\langle\eta_{n}, \xi_{n}\right\rangle \rightarrow\langle\eta, \xi\rangle=\int_{Y}\langle\hat{d}+\eta, \nabla \hat{u}+\xi\rangle \mathrm{d} y .
$$

Since, by Corollary 2.17, $\mathcal{B}^{n} \longmapsto \mathcal{B}$, Theorem 2.8 gives $(\nabla \hat{u}(y)+\xi, \hat{d}(y)+\eta) \in$ $B(y)$ for almost every in $Y$. Therefore $(\xi, \eta) \in A$. Noting $\xi+\eta=\zeta$, this implies $(\xi, \eta)=\left(J_{1}^{A} \zeta, A_{1} \zeta\right)$. Thus, one has $A_{1}^{n} \zeta \rightarrow A_{1} \zeta$ for every $\zeta \in \mathbf{R}^{N}$.

\subsection{Correctors}

In this section we first study the convergence $\int_{\Omega}\left\langle d_{\epsilon}-d_{0}, \nabla u_{\epsilon}-\nabla u_{0}\right\rangle \mathrm{d} x$ and then a corrector result.

Proposition 4.5. Under hypotheses of Theorem 4.1, assume there exists $\hat{u} \in L^{p}\left(\Omega ; W_{\text {per }}^{1, p}(\Omega)\right)$ and $\hat{d} \in L^{q}\left(\Omega \times Y ; \mathbf{R}^{N}\right)$ such that $\int_{Y} \hat{d} \mathrm{~d} y=0$ and a sequence $\left(\epsilon_{n}\right)$ with $\epsilon_{n} \rightarrow 0$

$$
\begin{cases}\mathcal{T}_{\epsilon_{n}}^{Y}\left(\nabla u_{\epsilon_{n}}\right) \rightarrow \nabla_{x} u_{0}+\nabla_{y} \hat{u} & \text { weakly in } L^{p}\left(\mathbf{R}^{N} \times Y ; \mathbf{R}^{N}\right), \\ \mathcal{T}_{\epsilon_{n}}^{Y}\left(d_{\epsilon_{n}}\right) \rightarrow d_{0}+\hat{d} & \text { weakly in } L^{q}\left(\mathbf{R}^{N} \times Y ; \mathbf{R}^{N}\right),\end{cases}
$$


then

$$
\int_{\Omega}\left\langle d_{\epsilon_{n}}-d_{0}-\mathcal{U}_{\epsilon_{n}}^{Y}(\hat{d}), \nabla u_{\epsilon_{n}}-\nabla u_{0}-\mathcal{U}_{\epsilon_{n}}^{Y}\left(\nabla_{y} \hat{u}\right)\right\rangle \mathrm{d} x \rightarrow 0 .
$$

Remark 24. Given a sequence $\left(\epsilon_{n}\right)$, the conditions of Proposition 4.5 are always satisfied up to a subsequence.

Proof. One has

$$
\begin{aligned}
& \int_{\Omega}\left\langle d_{\epsilon_{n}}-d_{0}-\mathcal{U}_{\epsilon_{n}}^{Y}(\hat{d}), \nabla u_{\epsilon_{n}}-\nabla u_{0}-\mathcal{U}_{\epsilon_{n}}^{Y}\left(\nabla_{y} \hat{u}\right)\right\rangle \mathrm{d} x= \\
& \frac{1}{|Y|} \int_{\mathbf{R}^{N} \times Y}\left\langle\mathcal{T}_{\epsilon_{n}}^{Y}\left(d_{\epsilon_{n}}\right)-\mathcal{T}_{\epsilon_{n}}^{Y}\left(d_{0}\right)-\mathcal{T}_{\epsilon_{n}}^{Y}\left(\mathcal{U}_{\epsilon_{n}}^{Y}(\hat{d})\right), \mathcal{T}_{\epsilon_{n}}^{Y}\left(\nabla u_{\epsilon_{n}}\right)-\mathcal{T}_{\epsilon_{n}}^{Y}\left(\nabla u_{0}\right)\right. \\
& \left.-\mathcal{T}_{\epsilon_{n}}^{Y}\left(\mathcal{U}_{\epsilon_{n}}^{Y}\left(\nabla_{y} \hat{u}\right)\right)\right\rangle \mathrm{d} x \mathrm{~d} y .
\end{aligned}
$$

The convergence then follows from (4.7), together with Theorems 3.5 and 3.15 .

As in [16] one can recover strong convergences as follows:

Theorem 4.6. Let $A_{\epsilon}, A_{0}$ be as in Theorem 4.1 and Proposition 4.5. If there exists $c>0, \beta \geq p$ and $\kappa_{\epsilon} \in L^{1}(\Omega)$ such that for almost every $x \in \Omega$, for every $\left(\xi_{1}, \eta_{1}\right),\left(\xi_{2}, \eta_{2}\right) \in A_{\epsilon}(x)$,

$$
\left\|\xi_{1}-\xi_{2}\right\|^{\beta} \leq c\left(\kappa_{\epsilon}(x)+\left\|\xi_{1}\right\|^{p}+\left\|\xi_{2}\right\|^{p}\right)^{(\beta / p)-1}\left\langle\eta_{1}-\eta_{2}, \xi_{1}-\xi_{2}\right\rangle .
$$

If $\sup _{\epsilon>0}\left\|\kappa_{\epsilon}\right\|_{L^{1}}<\infty$, then

$$
\nabla u_{\epsilon}-\mathcal{U}_{\epsilon}^{Y}\left(D_{y} \hat{u}\right) \rightarrow \nabla_{x} u_{0}
$$

strongly in $L^{p}(\Omega)$ as $\epsilon \rightarrow 0$.

Moreover, for every $\left(\xi_{1}, \eta_{1}\right),\left(\xi_{2}, \eta_{2}\right) \in A_{\epsilon}(x)$,

$$
\left\|\xi_{1}-\xi_{2}\right\|^{\beta} \leq c\left(\kappa_{0}(x)+\frac{\left\|\xi_{1}\right\|^{p}}{\alpha^{p}}+\frac{\left\|\xi_{2}\right\|^{p}}{\alpha^{p}}\right)^{(\beta-p) / p}\left\langle\eta_{1}-\eta_{2}, \xi_{1}-\xi_{2}\right\rangle,
$$

with

$$
\kappa_{0}(x)=\int_{Y} \liminf _{\epsilon \rightarrow 0} \mathcal{T}_{\epsilon}^{Y}\left(\kappa_{\epsilon}\right)(x, y) d y+\frac{2 p}{\alpha} \bar{m}(x) .
$$

Proof. We use the notations of Theorem 4.1. First note that by $(4.20),\left(u_{0}, \hat{u}\right)$ does not depend on the subsequence $\left(\epsilon_{n}\right)$. Therefore, the whole sequences converge: $u_{\epsilon} \rightarrow u$ in $W_{0}^{1, p}(\Omega)$ and $\mathcal{T}_{\epsilon}^{Y}\left(\nabla u_{\epsilon}\right) \rightarrow \nabla u_{0}+\nabla_{y} \hat{u}$ as $\epsilon \rightarrow 0$. Since $\mathcal{B}_{\epsilon} \longmapsto B$, there exists a sequence $\left(\xi_{\epsilon}, \eta_{\epsilon}\right) \in \mathcal{B}_{\epsilon}$ such that $\left(\xi_{\epsilon}, \eta_{\epsilon}\right) \rightarrow$ $\left(\nabla_{x} u_{0}+\nabla_{y} \hat{u}, d_{0}+\hat{d}\right)$ strongly in $L^{p}\left(\mathbf{R}^{N} \times Y ; \mathbf{R}^{N}\right) \times L^{q}\left(\mathbf{R}^{N} \times Y ; \mathbf{R}^{N}\right)$. 
By Hölder's inequality,

$$
\begin{aligned}
\int_{\mathbf{R}^{N} \times Y} & \left\|\mathcal{T}_{\epsilon}^{Y}\left(\nabla u_{\epsilon}\right)-\xi_{\epsilon}\right\|^{p} \mathrm{~d} x \mathrm{~d} y \\
\leq\left(\int_{\mathbf{R}^{N} \times Y} \frac{\left\|\mathcal{T}_{\epsilon}^{Y}\left(\nabla u_{\epsilon}\right)-\xi_{\epsilon}\right\|^{\beta}}{\left(\mathcal{T}_{\epsilon}^{Y}\left(\kappa_{\epsilon}\right)+\left\|\mathcal{T}_{\epsilon}^{Y}\left(\nabla u_{\epsilon}\right)\right\|^{p}+\left\|\xi_{\epsilon}\right\|^{p}\right)^{(\beta / p)-1}} \mathrm{~d} x \mathrm{~d} y\right)^{p / \beta} & \left(\int_{\mathbf{R}^{N} \times Y} \kappa_{\epsilon}+\left\|\mathcal{T}_{\epsilon}^{Y}\left(\nabla u_{\epsilon}\right)\right\|^{p}+\left\|\xi_{\epsilon}\right\|^{p} \mathrm{~d} x \mathrm{~d} y\right)^{1-p / \beta}
\end{aligned}
$$

By the boundedness $\mathcal{T}_{\epsilon}^{Y}\left(\nabla u_{\epsilon}\right)$ in $L^{p}\left(\Omega \times Y ; \mathbf{R}^{N}\right)$ and the inequality (4.20), there is $C>0$ such that

$$
\begin{aligned}
&\left(\int_{\mathbf{R}^{N} \times Y}\left\|\mathcal{T}_{\epsilon}^{Y}\left(\nabla u_{\epsilon}\right)-\xi_{\epsilon}\right\|^{p} \mathrm{~d} x \mathrm{~d} y\right)^{\beta / p} \\
& \leq C \int_{\mathbf{R}^{N} \times Y}\left\langle\mathcal{T}_{\epsilon}\left(d_{\epsilon}\right)-\eta_{\epsilon}, \mathcal{T}_{\epsilon}^{Y}\left(\nabla u_{\epsilon}\right)-\xi_{\epsilon}\right\rangle \mathrm{d} x \mathrm{~d} y
\end{aligned}
$$

Using successively the strong and weak convergences of $\left(\xi_{\epsilon}, \eta_{\epsilon}\right)$ and $\left(\mathcal{T}_{\epsilon}^{Y}\left(\nabla u_{\epsilon}\right), \mathcal{T}_{\epsilon}\left(d_{\epsilon}\right)\right)$ to $\left(\nabla_{x} u_{0}+\nabla_{y} \hat{u}, d_{0}+\hat{d}\right)$, and the convergence $(4.7)$, we have that

$$
\begin{aligned}
\lim _{\epsilon \rightarrow 0} & \int_{\mathbf{R}^{N} \times Y}\left\langle\mathcal{T}_{\epsilon}\left(d_{\epsilon}\right)-\eta_{\epsilon}, \mathcal{T}_{\epsilon}^{Y}\left(\nabla u_{\epsilon}\right)-\xi_{\epsilon}\right\rangle \mathrm{d} x \mathrm{~d} y \\
& =\lim _{\epsilon \rightarrow 0} \int_{\mathbf{R}^{N} \times Y}\left\langle\mathcal{T}_{\epsilon}\left(d_{\epsilon}\right), \mathcal{T}_{\epsilon}^{Y}\left(\nabla u_{\epsilon}\right)\right\rangle-\left\langle d_{0}+\hat{d}, \nabla_{x} u_{0}+\nabla_{y} \hat{u}\right\rangle \mathrm{d} x \mathrm{~d} y=0 .
\end{aligned}
$$

This implies $\left\|\mathcal{T}_{\epsilon}\left(D u_{\epsilon}\right)-\xi_{\epsilon}\right\| \rightarrow 0$. Since $\xi_{\epsilon} \rightarrow \nabla_{x} u_{0}+\nabla_{y} \hat{u}$ strongly in $L^{p}\left(\mathbf{R}^{N} \times Y\right)$, one has $\mathcal{T}_{\epsilon}^{Y}\left(\nabla u_{\epsilon}\right) \rightarrow \nabla_{x} u_{0}+\nabla_{y} \hat{u}$. Theorem 3.15 and Proposition 3.14 give the conclusion.

Since $\mathcal{T}_{\epsilon}^{Y}\left(A_{\epsilon}\right) \longmapsto B$, inequality 4.20 is satisfied with $\hat{\kappa}(x, y)=\liminf _{\epsilon \rightarrow 0} \mathcal{T}_{\epsilon}^{Y}\left(\kappa_{\epsilon}\right)(x, y)$ in place of $\kappa_{\epsilon}(x)$ for every $(\xi, \eta) \in B(x, y)$. For $i=1,2$, consider $\left(\xi_{i}, \eta_{i}\right) \in A_{0}$ and $\left(\hat{u}_{i}, \hat{d}_{i}\right)$ associated by definition (4.4). By Jensen's and Hölder's inequalities,

$$
\begin{aligned}
\left\|\xi_{1}-\xi_{2}\right\|^{\beta} & \leq\left(\frac{1}{|Y|} \int_{Y}\left\|\xi_{1}+\nabla \hat{u}_{1}(y)-\xi_{2}-\nabla \hat{u}_{2}(y)\right\|^{p} \mathrm{~d} y\right)^{\beta / p} \\
\leq & c \frac{1}{|Y|} \int_{Y} \frac{\left\|\xi_{1}+\nabla \hat{u}_{1}(y)-\xi_{2}-\nabla \hat{u}_{2}(y)\right\|^{\beta}}{\left(\hat{\kappa}+\left\|\xi_{1}+\nabla \hat{u}_{1}\right\|+\left\|\xi_{2}+\nabla \hat{u}_{2}\right\|^{p}\right)^{(\beta / p)-1}} \mathrm{~d} y \\
& \left(\frac{1}{|Y|} \int_{Y} \hat{\kappa}+\left\|\xi_{1}+\nabla \hat{u}_{1}\right\|^{p}+\left\|\xi_{2}+\nabla \hat{u}_{2}\right\|^{p} \mathrm{~d} y\right)^{(\beta / p)-1}
\end{aligned}
$$

The first factor on the right-hand side is controlled by inequality (4.20) for 
$B:$

$$
\begin{aligned}
& \frac{1}{|Y|} \int_{Y} \frac{\left\|\xi_{1}+\nabla \hat{u}_{1}(y)-\xi_{2}-\nabla \hat{u}_{2}(y)\right\|^{\beta}}{\left(\hat{\kappa}+\left\|\xi_{1}+\nabla \hat{u}_{1}\right\|^{p}+\left\|\xi_{2}+\nabla \hat{u}_{2}\right\|^{p}\right)^{(\beta / p)-1}} \mathrm{~d} y \\
& \quad \leq c \frac{1}{|Y|} \int_{Y}\left\langle\eta_{1}+\hat{d}_{1}(y)-\eta_{2}-\hat{d}_{2}(y), \xi_{1}+\nabla \hat{u}_{1}(y)-\xi_{2}-\nabla \hat{u}_{2}(y)\right\rangle \mathrm{d} y \\
& \quad=c\left\langle\eta_{1}-\eta_{2}, \xi_{1}-\xi_{2}\right\rangle .
\end{aligned}
$$

In order to bound the second factor, note that, by (2.6) and Young's and Jensen's inequalities,

$$
\begin{aligned}
& \frac{\alpha}{p} \frac{1}{|Y|} \int_{Y}\left\|\xi_{i}+\nabla \hat{u}_{i}\right\|^{p} \mathrm{~d} y \\
& \quad \leq\left\langle\eta_{i}, \xi_{i}\right\rangle-\frac{\alpha}{q} \frac{1}{|Y|} \int_{Y}\left\|\eta_{i}+\hat{d}_{i}\right\|^{p} \mathrm{~d} y+\bar{m}(x) \leq \frac{1}{p \alpha^{p-1}}\left\|\xi_{i}\right\|^{p}+\bar{m}(x) .
\end{aligned}
$$

Remark 25. Similarly, if there exists $C>0$ and $\beta \geq q$ such that for every $\left(\xi_{1}, \eta_{1}\right),\left(\xi_{2}, \eta_{2}\right) \in A_{\epsilon}(x)$

$$
\left\|\eta_{1}-\eta_{2}\right\|^{\beta} \leq c\left(\kappa_{\epsilon}(x)+\left\|\eta_{1}\right\|^{p}+\left\|\eta_{2}\right\|^{p}\right)^{(\beta-q) / q}\left\langle\eta_{1}-\eta_{2}, \xi_{1}-\xi_{2}\right\rangle,
$$

then

$$
d_{\epsilon}-\mathcal{U}_{\epsilon}^{Y}(\hat{d}) \rightarrow d_{0}
$$

strongly in $L^{q}(\Omega)$ as $\epsilon \rightarrow 0$.

Remark 26. The convergence $d_{\epsilon}-\mathcal{U}_{\epsilon}^{Y}(\hat{d}) \rightarrow d_{0}$ follows from Theorem 4.6 when $A_{\epsilon}$ and $A$ are single-valued by Proposition 2.7 and Lebesgue's dominated convergence Theorem. A similar result holds when $A_{\epsilon}$ and $A$ are injective and satisfy $(4.21), \nabla u_{\epsilon}-\mathcal{U}_{\epsilon}^{Y}\left(D_{y} \hat{u}\right) \rightarrow \nabla_{x} u_{0}$.

\section{Acknowledgment}

The authors wish to thank Professor Murat for helpfull discussion.

The second author was supported by a Research Fellow grant of the Belgian Fonds National de la Recherche Scientifique (FNRS). Part of this work was done while he was visting the Laboratoire J.-L. Lions (Université de Paris 6), supported by the Fonds Spéciaux de Recherche (Université catholique de Louvain). 


\section{References}

[1] H. Atтouch, Famille d'opérateurs maximaux monotones et mesurabilité, Annali di Matematica pura et applicata, IV, Vol CXX, (1979), 35-111.

[2] H. Aтtouch, Variational convergence for functions and operators, Applicable Mathematics Series, Pitman, Boston, Mass.-London, 1984.

[3] H. BREZIS, Opérateurs maximaux monotones et semi-groupes de contractions dans les espaces de Hilbert, North Holland Mathematics Studies, No. 5. Notas de Mathematica (50). North Holland Publishing Co., Amsterdam-London; American Elsevier Publishing Co. Inc., New York 1973.vi+183pp.

[4] F.E. BRowDER, Nonlinear variational inequalities and maximal monotone mappings in Banach spaces, Math. Ann. 183 (1969), 213-231.

[5] F.E. BRowDER, Nonlinear operators and nonlinear equations of evolution in Banach spaces, Nonlinear functional Analysis, Proc. Sympos. Pure Math. 18 Ameri. Math. Soc., 1976.

[6] C. Castaing and M. Valadier, Convex analysis and measurable multifunctions, Lecture Notes in Mathematics Studies, Vol. 580, Springer Verlag, 1977.

[7] V. Chiado'Piat, G. Dal Maso, and A. Defranceschi, G-convergence of monotone operators, C. Ann. Inst. H. Poincaré Anal. Non Linéaire 7 (3) (1990), 123-160.

[8] V. Chiado'Piat and G. Dal Maso, Homogenization of monotone operators, Nonlinear Anal. 14 (9) (1990), 717-732.

[9] D. Cioranescu, A. Damlamian, and G. Griso, Periodic unfolding and homogenization, C. R. Acad. Sci. Paris Math. 335 (2002), no. 1, 99-104.

[10] A. Damlamian, Convergence et dualité des fonctions convexes dans les espaces de Banach reflexifs, unpublished, 1975.

[11] A. Damlamian An elementary introduction to Periodic Unfolding, Multiscale problems and Asymptotic Analysis ; proceedings of the Midnig ht Sun Narvik Conference, June 2004, D. Lukkassen, A. Meidell and A. Piatnitski eds, GAKUTO International Series, Math.Sci. Appl. Vol. 24 (2005), Tokyo, p. 119-136.

[12] A. Damlamian, N. Meunier and J. Van Schaftingen, Periodic homogenization for normal convex integrands, to appear. 
[13] G. Francfort, F. Murat and L. TARTAR Monotone operators in divergence form with $x$-dependent multivalued graphs, Boll. U. M. I. 7 B (2004), 23-59.

[14] J. LERAY and J. L. Lions Quelques résultats de Visik sur les problemes elliptiques nonlinéaires par les méthodes de Minty-Browder, Bull. Soc. Math. France, 93 (1965), 97-107.

[15] N. Meunier and J. Van Schaftingen, Reiterated homogenization for elliptic operators, C. R. Acad. Sci. Paris, 340 (2005), no. 3, 209-214.

[16] N. Meunier and J. VAn Schaftingen, Reiterated homogenization for elliptic functions, J. Math. Pures Appl. 84 (2005), 1716-1743.

[17] G.J. Minty, Monotone (nonlinear) operators in Hilbert space, Duke Math. J. 29 (1962), 341-346.

[18] F. Murat and L. TarTar H-convergence, Topics in the Mathematical Modelling of Composite Materials (1997), 21-43.

[19] H. SCHAEFER Uber die Methode der a priori-Schranken, Math. Ann. 129 (1955), 415-416.

[20] L. Tartar, Cours Peccot, Collège de France, (1977).

[21] S. L. Trojanski, On locally uniformly convex and differentiable norms in certain non-separable Banach spaces, Studia Math. 37 (1970/71), 173180. 